(C) 2017. This manuscript version is made available under the CC-BY-NC-ND 4.0 license http://creativecommons.org/licenses/by-nc-nd/4.0/

DOI: 10.1016/j.freeradbiomed.2015.12.005

\title{
Unique cistrome defined as CsMBE is strictly required for Nrf2-sMaf
}

\section{heterodimer function in cytoprotection}

$$
\begin{aligned}
& \text { Akihito Otsuki }^{1} \text {, Mikiko Suzuki }{ }^{2}{ }^{*} \text {, Fumiki Katsuoka }{ }^{3} \text {, Kouhei Tsuchida }{ }^{1} \text {, Hiromi Suda }{ }^{1} \text {, } \\
& \text { Masanobu Morita }{ }^{1} \text {, Ritsuko Shimizu }{ }^{4} \text {, and Masayuki Yamamoto }{ }^{1,3, *}
\end{aligned}
$$

${ }^{1}$ Department of Medical Biochemistry, Tohoku University Graduate School of Medicine, Sendai 980-8575, Japan

${ }^{2}$ Center for Radioisotope Sciences, Tohoku University Graduate School of Medicine, Sendai 980-8575, Japan

${ }^{3}$ Tohoku Medical Megabank Organization, Tohoku University, Sendai 980-8573, Japan ${ }^{4}$ Department of Molecular Hematology, Tohoku University Graduate School of Medicine, Sendai 980-8575, Japan

\section{*Correspondence:}

Masayuki Yamamoto

Department of Medical Biochemistry, Tohoku University Graduate School of Medicine

2-1 Seiryo-machi, Aoba-ku, Sendai, Miyagi, 980-8575, Japan

Phone +81-22-717-8084; Fax +81-22-717-8090

E-mail: masiyamamoto@med.tohoku.ac.jp

Mikiko Suzuki

Center for Radioisotope Sciences, Tohoku University Graduate School of Medicine

2-1 Seiryo-machi, Aoba-ku, Sendai, Miyagi, 980-8575, Japan

Phone +81-22-717-8088; Fax +81-22-717-8090

E-mail: suzukimikiko@med.tohoku.ac.jp 


\section{Abstract}

32 Nrf2-small Maf (sMaf) heterodimer is essential for the inducible expression of

33 cytoprotective genes upon exposure to oxidative and xenobiotic stresses. While the

34 Nrf2-sMaf heterodimer recognizes DNA sequences referred to as the

35 antioxidant/electrophile responsive element (ARE/EpRE), we here define these DNA

36 sequences collectively as CNC-sMaf binding element (CsMBE). In contrast, large and small

37 Maf proteins are able to form homodimers that recognize the Maf recognition element

38 (MARE). CsMBE and MARE share a conserved core sequence but they differ in the

39 5'-adjacent nucleotide neighboring the core. Because of the high similarity between the

40 CsMBE and MARE sequences, it has been unclear how many target binding sites and target

41 genes are shared by the Nrf2-sMaf heterodimers and Maf homodimers. To address this issue,

42 we introduced a substitution mutation of alanine to tyrosine at position 502 in Nrf2, which

43 rendered the DNA-binding domain structure of Nrf2 similar to Maf, and generated knock-in

44 mice expressing the $\mathrm{Nrf}^{\mathrm{A} 502 \mathrm{Y}}$ mutant. Our chromatin immunoprecipitation-sequencing

45 analyses showed that binding sites of $\mathrm{Nrf2}^{\mathrm{A} 502 \mathrm{Y}}$-sMaf were dramatically changed from

46 CsMBE to MARE in vivo. Intriguingly, however, one-quarter of the $\mathrm{Nrf}^{\mathrm{A} 502 \mathrm{Y}}$-sMaf binding

47 sites also bound Nrf2-sMaf commonly and vice versa. RNA-sequencing analyses revealed

48 that $\mathrm{Nrf}^{\mathrm{A} 502 \mathrm{Y}}$-sMaf failed to induce expression of major cytoprotective genes upon stress

49 stimulation, which increased the sensitivity of $\mathrm{Nrf}^{\mathrm{A} 502 \mathrm{Y}}$ mutant mice to acute

50 acetaminophen toxicity. These results demonstrate that the unique cistrome defined as

51 CsMBE is strictly required for the Nrf2-sMaf heterodimer function in cytoprotection and 52 that the roles played by CsMBE differ sharply from those of MARE. 


\section{Highlights}

55 - Substitution of Ala-502 to Tyr renders the DNA-binding of Nrf2 similar to that of Maf

56 - Sequence recognition of $\mathrm{Nrf}^{\mathrm{A} 502 \mathrm{Y}}$ shifts from CsMBE to MARE

57 - $\quad \mathrm{Nrf2}^{\mathrm{A} 502 \mathrm{Y}}$ fails to induce major cytoprotective genes upon stress stimulation

58 - Recognition of CsMBE by Nrf2-sMaf is required for the cytoprotective function

59 - $\quad \mathrm{Nrf}^{\mathrm{A} 502 \mathrm{Y}}$ mutant mice are susceptible to oxidative and xenobiotic stresses

60

$61 \quad$ Keywords

62 Nrf2, Maf, CsMBE, MARE

63 


\section{Introduction}

65 Nrf2 (NF-E2-related factor 2) is a CNC (cap 'n' collar) family transcription factor that regulates inducible expression of an array of cytoprotective genes [1-3]. Nrf2 activates target genes in a stress-dependent manner through forming a heterodimer with small Maf proteins (sMaf). Under normal conditions, Nrf2 protein is constitutively trapped by Keap1 (Kelch-like ECH-associated protein 1) and is degraded through the proteasome pathway in the cytoplasm $[4,5]$. Oxidative and electrophilic stresses inactivate Keap1 and stabilize Nrf2

[3]. The stabilized Nrf2 is translocated into nucleus and activates expression of target genes that encode enzymes/proteins scavenging of reactive oxygen species (ROS) or related to detoxification of xenobiotics and drug metabolism.

The CNC family transcription factors, including NF-E2 p45, Nrf1, Nrf2, Nrf3, Bach1 and Bach2, form heterodimers with the sMaf family of transcription factors, MafF, MafG and MafK [6-9]. The CNC-sMaf heterodimers bind to a consensus DNA sequences, which are called various names, such as antioxidants response element (ARE) [10,11], electrophile response element (EpRE) [12], and NF-E2 binding element [13], via their basic region-leucine zipper (bZip) structure. We have compared these binding sequences and found that they show a common consensus sequence, 5'-(A/G)TGA(G/C)nnnGC-3', but these recognition elements are partially distinct from the element bound by Maf homodimers. Therefore, in this study we refer to the sequence recognized by CNC-sMaf, including the ARE, the EpRE and the NF-E2 binding element, as $\underline{\mathrm{CNC}}$-sMaf binding element (CsMBE). Of note, the CsMBE sequence shares substantial overlap with that of the Maf recognition element (MARE), a palindromic motif 5'-TGCTGA(G/C)TCAGCA-3' (underline shows overlapping sequence with CsMBE) that binds homodimers of large Maf proteins (c-Maf, MafA/L-Maf, MafB and Nrl) and sMaf proteins [14-16]. Because of the significant overlap, there has been substantial confusion in the cistrome dynamics or the binding sequence 
89 selection by the CNC-sMaf heterodimers and Maf homodimers.

CsMBE and MARE harbor TRE (phorbol 12-O-tetradecanoate-13-acetate

91 (TPA)-responsive element; TGA(G/C)TCA) or binding site for AP-1 in the middle of the motifs [17]. MARE harbors GC at the 5' of TRE, while CsMBE retains A/G at the position.

93 It is interesting to note that the presence of a GC dinucleotide adjacent to the TRE stabilizes

94 MafG homodimer binding $[11,15,16]$. Indeed, the surface plasmon resonance (SPR)-based protein-DNA interaction studies revealed that the GC sequence is essential for recognition by sMaf proteins [18]. Structural analysis of MafG revealed that Arg-57, Asp-61 and Tyr-64 of the basic region of MafG are important for the recognition of the GC sequence $[19,20]$. their ancestors [21-24].

While Arg-57 and Asp-61 of MafG are conserved in the basic region of the CNC family

101 proteins, the residue of Nrf2 corresponding to Tyr-64 of MafG is converted to alanine 102 residue (Ala-502). The alanine residue is highly conserved among the CNC family 103 transcription factors (Nrf1, Nrf2, Nrf3, NF-E2 p45, Bach1 and Bach2) and ancestors of CNC 104 family proteins; SKN-1 (Skinhead family member-1) in Caenorhabditis elegans [25], CncC in Drosophila melanogaster [26] and Nfe212a in Danio rerio [27, 28]. We and others

106 previously found that the alanine and tyrosine in the basic region are critical residues to 107 determine the unique binding preference of Nrf2-sMaf heterodimer and Maf homodimer to 108 CsMBE and MARE, respectively [29, 30]. A heterodimer of an Nrf2 mutant generated by 109 replacing the Ala-502 residue with a tyrosine residue ( $\left.\mathrm{Nrf}_{2}^{\mathrm{A} 502 \mathrm{Y}}\right)$ and MafG displays binding 110 preferences similar to MafG homodimer [29]. Since both $\mathrm{Nrf2}^{\mathrm{A} 502 \mathrm{Y}}$ and MafG require the $111 \mathrm{GC}$ sequence in the TRE flanking region, the $\mathrm{Nrf}^{\mathrm{A} 502 \mathrm{Y}}$-sMaf heterodimer displays similar 112 high-affinity binding to the palindromic MARE to that of MafG homodimer.

113 To assess the contribution of CsMBE recognition by Nrf2-sMaf to cytoprotective 
114 function, we generated $\mathrm{Nrf}^{\mathrm{A} 502 \mathrm{Y}}$ mutant knock-in mice using a genome-editing technique.

115 Utilizing peritoneal macrophages from the $\mathrm{Nrf}^{\mathrm{A} 502 \mathrm{Y}}$ mutant knock-in mice, we performed 116 comprehensive analyses of $\mathrm{Nrf} 2$ binding sites [chromatin immunoprecipitation 117 (ChIP)-sequencing (ChIP-Seq)] and gene expression profiles [RNA-sequencing (RNA-Seq)]. 118 To our surprise, we found that the $\mathrm{Nrf} 2^{\mathrm{A} 502 \mathrm{Y}}$ mutant fails to support the expression of 119 three-quarters of the electrophile-inducible cytoprotective genes, including glutathione 120 conjugation- and hydrogen peroxide degradation-related enzyme genes, inducible expression 121 of which are normally supported by wild-type Nrf2. Meanwhile, the $\mathrm{Nrf2}^{\mathrm{A} 502 \mathrm{Y}}$ mutant still 122 retains the ability to support the expression of one-quarter of the electrophile-inducible genes 123 under the Nrf2 regulation. These results thus unequivocally demonstrate that CsMBE and 124 MARE, binding sequences for CNC-sMaf heterodimer and Maf homodimer, respectively, 125 generate distinct sets of gene regulations. Specific recognition of the CsMBE by the 126 Nrf2-sMaf heterodimer is critical for the inducible expression of Nrf2 target genes, which 127 play key roles in the cytoprotection against ROS and toxic electrophiles. 
130 Generation of $\boldsymbol{N r f 2 ^ { A 5 0 2 Y }}$ knock-in mice. A plasmid expressing single-guide RNA (sgRNA) 131 and Cas9 was constructed as described previously [31]. Plasmid vector pX330 [32] 132 expressing Cas9 and gRNA was digested with BbsI and a pair of oligo DNA recognizing 133 Nrf2 targeting site (5'-AAG TCG CCG CCC AGA ACT GT-3') was ligated to the linealized 134 vector. Donor oligo DNA encoding substitution from alanine to tyrosine was designed as follows; 5'-ATC CGA GAT ATA CGC AGG AGA GGT AAG AAT AAA GTC TAC GCC

136 CAG AAC TGT AGG AAA AGG AAG CTG GAG-3'. The plasmid and donor DNA were 137 co-injected into BDF1 fertilized eggs. We obtained two lines of $N r f 2^{A 502 Y}$ knock-in mice. All 138 mice were handled according to Regulations for Animal Experiments and Related Activities 139 at Tohoku University.

141 Mouse genotyping. Genomic DNA was extracted from a piece of tail. The DNA samples 142 were genotyped by using TaqMan SNP Genotyping Assay System (Applied Biosystems). 143 Wild type (WT) alleles were detected by 2'-chloro-7'-phenyl-1, 144 4-dichliro-6-carboxyfluorescein (VIC)-labeled probes, and mutant alleles were detected by 145 6-carboxyfluorescein (FAM)-labeled probes.

147 Genomic DNA and cDNA sequencing. Genomic DNA was extracted from a piece of tail. 148 RNA extractions from brain, thymus, lung, heart, liver, pancreas, spleen, kidney, esophagus, 149 skeletal muscle were conducted for cDNA synthesis. Targeted region of Nrf2 gene was 150 amplified by PCR using following primers; forward, 5'- AAG ACA AAC ATT CAA GCC 151 GC-3'; reverse, 5'- GCT TTT GGG AAC AAG GAA CA-3'. The amplicon was sequenced 152 using ABI 3100 sequencer. The primer sequence for sequence was 5'-GCT TTT GGG AAC AAG GAA CA-3'. 
155 Peritoneal macrophage isolation and cell culture. 7-8 weeks of mice were received an

156 intraperitoneal injection of $4 \%$ thioglycolate broth. Four days later, macrophages collected 157 by intraperitoneal lavage were cultured in RPMI 1640 medium containing $10 \%$ fetal bovine 158 serum and 1\% penicillin-streptomycin [33]. For analysis of Nrf2-induced state, the 159 macrophages were treated with $100-\mu \mathrm{M}$ diethylmaleate (DEM). To test the cell viability 160 after 12 hours of menadione treatment, Cell Count Reagent SF (nacalai tesque) was used. 161 DEM and menadione were from Wako Pure Chemicals and Sigma-Aldrich, respectively.

163 Flow cytometry analysis. The cells were stained with antibodies to Gr-1, Mac1, and F4/80 164 conjugated with FITC, APC, and PE, respectively. These antibodies were from eBioscience. 165 The stained cells were analyzed with FACSCanto II and the data analyses were performed 166 with FlowJo software (Tree Star). RNA extraction and quantitative RT-PCR. RNA was extracted with Sepasol-RNA I 169 Super G (nacalai tesque) and reverse-transcribed with ReverTra Ace qPCR RT Master Mix 170 with gDNA Remover (TOYOBO) according to the manufacturer's instruction. Quantitative 171 PCR was run on ABI7300 (Applied Biosystems). We used the following primers and probe 172 to detect mRNA levels; Nrf2, forward primer, 5'-CAA GAC TTG GGC CAC TTA AAA 173 GAC-3'; reverse primer, 5'-AGT AAG GCT TTC CAT CCT CAT CAC-3'; probe 5'-AGG 174 CGG CTC AGC ACC TTG TAT CTT GA-3', 18S rRNA, forward primer, 5'-CGG CTA 175 CCA CAT CCA AGG AA-3'; reverse primer, 5'-GCT GGA ATT ACC GCG GCT-3'; and 176 Taqman probe, 5'-TGC TGG CAC CAG ACT TGC CCT C-3'.

178 Immunoblot analyses. Peritoneal macrophages were treated with $100-\mu \mathrm{M}$ DEM for 3 hours. 
179 Nuclear lysate for immunoblot was prepared using NE-PER Nuclear and Cytoplasmic

180 Extraction Reagents (ThermoFisher Scientific). $5 \mu \mathrm{g}$ of nuclear lysate was subjected to 181 immunoblot using anti-Nrf2 [34] and anti-Lamin B (M-20) (Santa-Cruz; sc-6217) antibodies. 182 The densitometries of image were analyzed with ChemiDoc MP Imaging System (Bio-Rad), 183 and normalized to Lamin B intensity.

185 ChIP-Seq analysis. For ChIP-Seq analysis, the peritoneal macrophages were treated with $186 \quad 100-\mu \mathrm{M}$ DEM for 4 hours as described [11] with minor modifications. ChIP was performed 187 with anti-Nrf2 antibody (Cell Signaling Technology; D1Z9C). DNA libraries were prepared 188 from 1.5 or $2 \mathrm{ng}$ of ChIP and input samples quantified with Qubit Fluorometer (Life 189 Technologies), using Mondrian SP+ and Ovation SP Ultralow DR Multiplex System 190 (TaKaRa). The constructed libraries were amplified by PCR and DNA fragments in 300-600 191 bp in size were yielded with AMPure XP Kit (BECKMAN COULTER). Prepared samples 192 were quantified by quantitative MiSeq (qMiSeq) method [35], followed by high throughput 193 sequencing using HiSeq2500 (Illumina) to generate 101 base-single reads. Three biological 194 replicates of ChIPed DNA and Input DNA prepared from each genotype and ChIP-Seq 195 analyses were conducted with these samples.

197 ChIP-Seq data analyses. The sequenced reads were mapped to the mouse genome (mm9) 198 using Bowtie2 software [36]. The mapped tags were visualized by using Integrative 199 Genomics Viewer [37]. Peak calling was performed using a model-based analysis of 200 ChIP-seq (MACS) version 1.4.2 [38]. DNA motif construction was performed using 201 MEME-ChIP version 4.10.0 [39]. Extraction of ARE motifs was performed using R based 202 script. 
RNA-Seq analysis. Total RNA was prepared by using RNeasy Mini Kit (QIAGEN) and 1.5

205 $\mu \mathrm{g}$ of total RNA was used for further steps. Isolation of poly(A)-tailed RNA and library construction were performed using Sureselect Strand Specific RNA Sample Prep Kit (Agilent Technologies). The libraries were sequenced using NextSeq500 (Illumina) for 86 cycles of single read. Three biological replicates were performed in each genotype.

RNA-Seq data analyses. TopHat [40] was used for mapping of RNA-Seq data, and Cufflinks version 2.1.1 [41] was used for quantifying the expression level of each gene as fragments per kilobase of exon per million fragments (FPKM) with default parameters. The differentially expressed genes were identified using Cuffdiff version 2.1.1, threshold of $q$ value $<0.05$. The KEGG pathway analysis was performed using DAVID Bioinformatics Resource 6.7 (http://david.abcc.ncifcrf.gov/). The KEGG pathway significantly enriched were defined as $p$ value $<0.05$. The $p$ values were corrected using Benjamini-Hochberg procedure. The gene set analysis was performed using the Gene Set Enrichment Analysis (GSEA) software [42]. The gene set was created by using data described in references [11, 43].

Acetaminophen (APAP) induced liver injury model. Following 16-hours fasting, 10-12 weeks male mice were treated with $125-\mathrm{mg} / \mathrm{kg}$ or $200-\mathrm{mg} / \mathrm{kg}$ APAP by intraperitoneal injection and sacrificed 6 hours after dosing. APAP was purchased from Sigma-Aldrich. Using blood serum obtained from posterior vena cava of anesthetized animal, plasma alanine transaminase (ALT) and asparate transaminase (AST) were determined using FUJI DRI-CHEM 7000V (FUJIFILM). Liver sample were fixed in 10\% formalin solution and stained with hematoxylin and eosin (HE). 
229 Accession number. The data discussed in this publication have been deposited in NCBI's 230 Gene Expression Omnibus [44] and are accessible through GEO Series accession number 231 GSE75177 (https://www.ncbi.nlm.nih.gov/geo/query/acc.cgi?acc=GSE75177). 


\section{Results}

234 Generation of $\operatorname{Nrf}^{\boldsymbol{A 5 0 2 Y}}$ knock-in mice. Dimetric transcription factors that contain Maf 235 protein can bind various cis-acting element sequences. Whilst Maf homodimers recognize 236 MARE sequences, CNC-sMaf heterodimers recognize CsMBE (Fig. 1A). Molecular basis of 237 this cis-element selection resides in the structural difference in Maf and CNC transcription 238 factors, and substituting an amino acid residue modifies this specificity. Substitution of Nrf2 239 Ala-502 residue to tyrosine brings in a significant difference in cistrome, and $\mathrm{Nrf}^{\mathrm{A} 502 \mathrm{Y}}$ 240 becomes recognizing Maf-oriented sequence. Accordingly, the recognition sequence 241 specificity of Nrf2-sMaf heterodimer changes from CsMBE to MARE [29] (Figs. 1A and 242 1B). However, in biological context in vivo, the importance of Nrf2 binding specificity to 243 CsMBE has not been fully evaluated.

244 To examine how germline modification to $\mathrm{Nrf2}^{\mathrm{A} 502 \mathrm{Y}}$ influences the Nrf2-sMaf activity, 245 we generated $\mathrm{Nrf}^{A 502 Y}$ knock-in mice using the CRISPR/Cas9 technology. To this end, we 246 designed a guide RNA (gRNA) containing 20 nucleotides capable of recognizing the $N r f 2$ 247 target site followed by a protospacer adjacent motif (PAM) to recruit Cas9 to the target site 248 (Fig. 1C). We generated a plasmid expressing both Cas9-encoding mRNA and the gRNA 249 [31]. We next introduced 69-mer oligo-DNA including mutations from GCC to TAC 250 resulting in substitution of the 502nd alanine to tyrosine (A502Y) for homologous 251 recombination. We co-injected both plasmid and oligo-DNA into fertilized eggs. We 252 obtained 24 pups. To verify homologous recombination of genomic DNA, we sequenced the 253 targeted regions, and identified two pups carrying mono-allelic A502Y mutation. We then 254 crossed these $\mathrm{Nrf}^{\mathrm{A} 502 \mathrm{Y}}$ founder mice with wild-type mice and established two lines of 255 knock-in substitution mice. Through genomic DNA sequencing analyses, we confirmed both 256 TAC (encoding tyrosine) and GCC (encoding alanine) in the heterozygous (Nrf $2^{A Y /+}$ ) 257 offspring (Fig. 1D, middle panel). 
To examine whether $\mathrm{Nrf}^{\mathrm{A} 502 \mathrm{Y}}$ is expressed in the $\mathrm{Nrf}^{A Y /+}$ mice, we prepared RNA samples from various tissues of the $\mathrm{Nrf}^{A Y /+}$ mice, and synthesized Nrf2 cDNA and sequenced. We detected comparable level of TAC and GCC in all the tissues of the $\mathrm{Nrf}^{A Y /+}$ mice examined, indicating successful homologous recombination of $\mathrm{Nrf}^{\mathrm{A} 502 \mathrm{Y}}$ (Fig. 1E). We further crossed $N r f 2^{A Y /+}$ mice and obtained homozygous $\left(N r f 2^{A Y / A Y}\right.$ ) mice (Fig. 1D, lower panel). Body-weight-gain of both male and female $N r f 2^{A Y / A Y}$ mice is comparable with that of the wild-type mice (Fig. 1F) and the mice were fertile.

\section{$N r 2^{A Y / A Y}$ macrophages are more susceptible to the cytotoxic effect of xenobiotics. To} examine whether $N r f 2^{A Y / A Y}$ mice preserves cytoprotective activities assisted by $\mathrm{Nrf}$, we employed the thioglycolate-elicited peritoneal macrophage system [33]. We injected thioglycolate into $\mathrm{Nrf}^{+/+}$and $\mathrm{Nrf}^{A Y / A Y}$ mice and harvested peritoneal macrophages (Fig. 2A). Almost all cells obtained from both $\mathrm{Nrf}^{+/+}$and $N r f 2^{A Y / A Y}$ peritoneal lavage exhibited $\mathrm{Mac}^{+} \mathrm{Gr} 1^{-} \mathrm{F} 4 / 80^{+}$surface markers, indicating that macrophage induction was comparable between $N r f 2^{+/+}$and $N r f 2^{A Y / A Y}$ mice (Fig. 2B).

We next treated the peritoneal macrophages, harvested both from $\mathrm{Nrf}^{+/+}$and $\mathrm{Nrf} 2^{A Y / A Y}$ mice, with an electrophilic Nrf2 inducer DEM (Fig. 2A). We found that the Nrf2 mRNA level of $N r f 2^{A Y / A Y}$ macrophages was comparable to that of wild type under the basal and DEM-induced conditions (Fig. 2C). Furthermore, comparable level of Nrf2 protein was accumulated in the nucleus under the DEM-treated condition in the $\mathrm{Nrf}^{+/+}$and $\mathrm{Nrf} 2^{A Y / A Y}$ macrophages (Figs. 2D and 2E). These results indicate that stress-responsiveness of $N r f 2^{A Y / A Y}$ macrophages were not significantly different from that of $\mathrm{Nrf2}^{+/+}$, regarding intercellular Nrf2 localization and the abundance.

To test cytoprotective function of $\mathrm{Nrf}^{\mathrm{A} 502 \mathrm{Y}}$, we then examined susceptibility of $\mathrm{Nrf} 2^{A Y / A Y}$ macrophages against menadione, which is a free radical-generating compound and is 
283 well-established stressor for testing roles played by Nrf2 in the oxidative stress response [45].

284 Cell viabilities of the $N r f 2^{A Y / A Y}$ macrophages were lower than those of the $N r f 2^{+/+}$ 285 macrophages (Fig. 2F), indicating that the $\operatorname{Nrf}^{A Y / A Y}$ macrophages were more susceptible to 286 toxicity of xenobiotics than the $\mathrm{Nrf} 2^{+/+}$macrophages.

288 ChIP-Seq analyses of $\mathrm{Nrf2}$ and $\mathrm{Nrf}^{\mathrm{A502Y}}$ reveal their preference of binding sequences.

289 Since the $N r f 2^{A Y / A Y}$ macrophages were more susceptible to toxicity of xenobiotics, we 290 assumed that $\mathrm{Nrf}^{\mathrm{A} 502 \mathrm{Y}}$ might fail to recognize CsMBE in the regulatory regions of Nrf2 291 target genes and therefore fail to induce their expression. To confirm preferences of binding 292 sequences of $\mathrm{Nrf} 2$ and $\mathrm{Nrf}^{\mathrm{A} 502 \mathrm{Y}}$ in vivo, we performed ChIP-Seq analyses using an 293 anti-Nrf2 antibody on the DEM-treated peritoneal macrophages derived from $\mathrm{Nrf} 2^{+/+}$and $294 \mathrm{Nrf}^{A Y / A Y}$ mice. The ChIP-Seq analyses were performed using three biological replicates 295 from each genotype. We defined $\mathrm{Nrf} 2$ and $\mathrm{Nrf}^{\mathrm{A} 502 \mathrm{Y}}$ binding peaks as peaks called in three 296 or two samples in the three replicates [46]. We obtained 1062 peaks for Nrf2 binding sites 297 and 1304 peaks for Nrf2 ${ }^{\mathrm{A} 502 \mathrm{Y}}$ binding sites (Fig. 3A). Of the $1062 \mathrm{Nrf2}$ binding sites, 669 298 peaks were recognized only by Nrf2. We thus designated the 669 sites as "WT-specific" 299 sites. Meanwhile, we identified 911 peaks to which only $\mathrm{Nrf2}^{\mathrm{A} 502 \mathrm{Y}}$ bound. We named these 300911 sites as "AY-specific" sites. Of these Nrf2 and Nrf2 ${ }^{\mathrm{A} 502 \mathrm{Y}}$ peaks, 393 peaks overlapped 301 between both $\mathrm{Nrf} 2$ and $\mathrm{Nrf2}^{\mathrm{A} 502 \mathrm{Y}}$. We designated the 393 sites that both $\mathrm{Nrf2}$ and $\mathrm{Nrf} 2^{\mathrm{A} 502 \mathrm{Y}}$ 302 bound to as "Common" sites. Typical peak profiles for Nrf2, Nrf2 $2^{\mathrm{A} 502 \mathrm{Y}}$ and the overlap of $303 \mathrm{Nrf2}$ and $\mathrm{Nrf}^{\mathrm{A} 502 \mathrm{Y}}$ are shown in Figure 3B. We found that the Nrf2 binding to the common 304 sites showed higher probability of binding than that of WT- and AY-specific sites, 305 suggesting that $\mathrm{Nrf2}$ and $\mathrm{Nrf}^{\mathrm{A} 502 \mathrm{Y}}$ binding to Common sites is tighter than that to WT- and 306 AY-specific sites (Fig. 3C). These results thus demonstrate that the alanine to tyrosine 307 substitution of Nrf2 502 position elicits marked conformation change, so that target-binding 
sites of $\mathrm{Nrf2}{ }^{\mathrm{A} 502 \mathrm{Y}}$ in vivo are largely different from those of wild-type Nrf2.

To determine consensus binding motifs for $\mathrm{Nrf}^{\mathrm{A} 502 \mathrm{Y}}$ and $\mathrm{Nrf2}$ in WT-specific, Common and AY-specific sites, we extracted sequences within \pm 150 bp of each peak center and performed de novo motif analysis. Core sequences of TRE (position 1-7) neighbored by 3' GC motif (position 8 and 9) appeared to be similar in WT-specific, Common and AY-specific sites (Fig. 3D). Consistent with our previous report [11], nucleotides A or G (A/G) at 5'-end neighboring to TRE core sequence (position 0) was enriched in WT-specific sites $\left(E\right.$-value $\left.=5.8 \times 10^{-724}\right)$, conforming our original observation that $\mathrm{Nrf2}$ recognizes CsMBE. On the other hand, the most enriched nucleotide at position 0 of AY-specific sites was $\mathrm{C}$, showing that the binding preference of $\mathrm{Nrf}^{\mathrm{A} 502 \mathrm{Y}}$ mimics that of sMaf homodimer or MARE in vivo $\left(\right.$ E-value $\left.=2.5 \times 10^{-521}\right)$. Of note, we did not detect enrichment of a specific base at position 0 in Common sites by de novo motif analysis $\left(E\right.$-value $\left.=1.2 \times 10^{-365}\right)$.

To analyze the nucleotide at position 0 in detail, we extract core motifs (position 1-9) within \pm 150 bp of each peak center and examined frequency of bases at position 0 . Nucleotides A/G and $\mathrm{C}$ were enriched at position 0 of TRE in WT-specific and AY-specific sites, respectively, showing a good agreement with de novo motif analysis (Fig. 3E). On the other hand, we found that $\mathrm{A}, \mathrm{G}$, or $\mathrm{C}$ but not $\mathrm{T}$ were enriched at position 0 in Common sites, showing that Common sites exhibit DNA preference of either WT-specific or AY-specific sites at position 0 (Fig. 3E).

A number of previous papers show that TMA sequence located the $5^{\prime}$ side of the CsMBE (position -5 to -3 in Fig. 3F, M represents A or C) influences activation of genes containing the element $[11,47-50]$. Therefore, we examined prevalence of the TMA motif in 5' region of WT-specific, Common and AY-specific sites. We found that the TMA-motif, especially TCA-motif, was observed in $6.9 \%$ and $8.0 \%$ of CsMBE of WT-specific and Common sites, respectively. On the other hand, TMA sequence was not enriched in AY-specific sites (3.0\% 
333 of motif, Fig. 3F). These results suggest that TCA at position -5 to -3 may support the

334 binding of Nrf2-sMaf heterodimer to CsMBE but not support the binding of Maf homodimer 335 to MARE in vivo.

337 Impairment of transcriptional activity in $\mathbf{N r f 2}^{\mathbf{A 5 0 2}}$ macrophages. Since the preference 338 of binding sequences of $\mathrm{Nrf}^{\mathrm{A} 502 \mathrm{Y}}$ shifted from CsMBE to MARE, it is expected that $339 \mathrm{Nrf2}^{\mathrm{A} 502 \mathrm{Y}}$ might support expression of a distinct gene set from that supported by Nrf2. To 340 examine this issue, we performed RNA-Seq analysis and compared gene expression profiles 341 in peritoneal macrophages from $\mathrm{Nrf}^{+/+}$and $\mathrm{Nrf}^{A Y / A Y}$ mice between basal and DEM-induced 342 conditions. We found that expression levels of 1402 genes were significantly changed upon 343 DEM stimulation in the $\mathrm{Nrf}^{+/+}$macrophages, in which 696 genes were upregulated and 706 344 genes were downregulated (Fig. 4A). On the other hand, we found that expression levels of 345402 genes were changed upon DEM stimulation in $\mathrm{Nrf}^{A Y / A Y}$ macrophages, in which 148 346 genes were upregulated and 254 genes were downregulated (Fig. 4B). Thus, the numbers of 347 upregulated and downregulated genes were strikingly decreased in $N r f 2^{A Y / A Y}$ macrophages 348 compared to those in $\mathrm{Nrf2}^{+/+}$macrophages.

349 Furthermore, the majority (309 out of 402 genes) of upregulated and downregulated 350 genes [referred to as differentially expressed genes (DEGs)] in $N r f 2^{A Y / A Y}$ macrophages 351 overlapped with DEGs in the $\mathrm{Nrf}^{+/+}$macrophages (Figs. 4C). As shown in Figure 4D, we 352 identified 1093 DEGs (586 and 507 genes were upregulated and downregulated, 353 respectively) observed only in the $\mathrm{Nrf}^{+/+}$macrophages (WT-specific DEGs), 309 DEGs 354 (110 and 199 genes were upregulated and downregulated, respectively) observed in both $355 \mathrm{Nrf}^{+/+}$and $\mathrm{Nrf} 2^{A Y / A Y}$ macrophages (Common DEGs), 93 DEGs (38 and 55 genes were 356 upregulated and downregulated, respectively) observed only in the $N r f 2^{A Y / A Y}$ macrophages 357 (AY-specific DEGs). The number of WT-specific DEGs was much larger than those of 
358 common and AY-specific DEGs. These results unequivocally demonstrate that $\mathrm{Nrf}^{\mathrm{A} 502 \mathrm{Y}}$

359 lacks the induction and repression abilities for the majority of Nrf2 target genes.

360 To annotate upregulated and downregulated genes in the $\mathrm{Nrf}^{+/+}$and $\mathrm{Nrf} 2^{A Y / A Y}$ 361 macrophages, we performed a KEGG pathway analysis. We found that known

362 Nrf2-dependent pathways such as glutathione metabolism and pentose phosphate pathway were enriched in genes upregulated specifically in the $N r f 2^{+/+}$macrophages (Fig. 4E). On the

364 other hand, inflammation-related pathways such as chemokine signaling pathway, focal

365 adhesion and leukocyte transendothelial migration pathways were enriched in the gene group 366 specifically downregulated in the $\mathrm{Nrf}^{+/+}$macrophages, showing very good agreement with 367 the recent findings that Nrf2 regulates anti-inflammatory genes [51, 52].

369 Nrf2 $^{\mathbf{A 5 0 2 Y}}$ fails to induce major cytoprotective genes. Since known Nrf2-dependent 370 pathways were enriched in the gene set upregulated specifically in the $\mathrm{Nrf}^{+/+}$macrophages, 371 we next examined whether Nrf2 ${ }^{\mathrm{A} 502 \mathrm{Y}}$ failed to induce known Nrf2 target genes. Our gene set 372 enrichment analysis (GSEA) showed that differentially expressed genes only in the $\mathrm{Nrf2}^{+/+}$ macrophages contained known Nrf2 target genes (Fig. 5A). We found that expression levels of genes related to quinone detoxification (Nqol), glutathione (GSH) conjugation (Gstml and Gstp1), GSH synthesis (Gss, Gclm and Gclc), GSH reduction (Gsr), hydrogen peroxide degradation (Cat), and pentose phosphate pathway (Taldol) were induced specifically in the $377 \mathrm{Nrf}^{+/+}$macrophages (Fig. 5B). In contrast, DEM induction of these genes was abrogated 378 almost completely in the $\mathrm{Nrf}^{\mathrm{A} 502 \mathrm{Y}}$ macrophages. Expression levels of genes related to heme degradation (Hmoxl), transcription factor (Mafg), and autophagy (Sqstm1) were induced both in the $\mathrm{Nrf}^{+/+}$and $\mathrm{Nrf} 2^{A Y / A Y}$ macrophages. These results indicate that $\mathrm{Nrf} 2^{\mathrm{A} 502 \mathrm{Y}}$ lost 381 ability to induce major cytoprotective genes.

382 To assess whether $\mathrm{Nrf} 2$ and $\mathrm{Nrf}^{\mathrm{A} 502 \mathrm{Y}}$ directly regulate these genes, we examined 
383 binding peaks of Nrf2 and $\mathrm{Nrf2}^{\mathrm{A} 502 \mathrm{Y}} \mathrm{ChIP-Seq}$ in the proximity of these genes. Expectedly, 384 the genes that were induced specifically in the $\mathrm{Nrf2}^{+/+}$macrophages, including $\mathrm{Nqo1}$, Gclm, 385 Gss and Cat, harbored WT-specific peaks (Fig. 5D). In addition, the genes that were induced 386 both in the $\mathrm{Nrf}^{+/+}$and $\mathrm{Nrf} 2^{A Y / A Y}$ macrophages, including Mafg, Hmoxl and Sqstm 1, harbored 387 Common peaks (Fig. 5E). These results support our contention that differences in the 388 Nrf2-sMaf cistrome indeed affect the gene expression profiles.

CsMBE recognition of Nrf2-sMaf is required for liver protection from APAP toxicity.

391 To examine whether $\mathrm{Nrf} 2^{\mathrm{A} 502 \mathrm{Y}}$ mutant mice are more susceptible to toxicity than wild-type 392 mice, we finally examined susceptibility of $N r f 2^{+/+}$and $N r f 2^{\mathrm{AY} / \mathrm{AY}}$ mice to acetaminophen 393 (APAP) toxicity. We intraperitoneally administered low-dose (125 mg/kg) and high-dose $394(200 \mathrm{mg} / \mathrm{kg})$ APAP to $N r f 2^{+/+}$and $N r f 2^{\mathrm{AY} / \mathrm{AY}}$ mice, which were fasted for 16 hours 395 beforehand. We analyzed these mice 6-hours after re-feeding (Fig. 7A).

396 Levels of liver damage indicators, ALT and AST, in the $N r f 2^{\mathrm{AY} / \mathrm{AY}}$ mice were 397 significantly higher than those in the $\mathrm{Nrf2}^{+/+}$mice in the low-dose examination (Fig. 7B). 398 While there were some fluctuations perhaps due to toxicity in the high-dose examination, the 399 results showed reproducibility. Histological analysis revealed that liver damage in the $400 N r f 2^{\mathrm{AY} / \mathrm{AY}}$ mice was more severe than those in the $N r f 2^{+/+}$mice (Fig. 7C). These results thus 401 demonstrate that the $\mathrm{Nrf}^{\mathrm{A} 502 \mathrm{Y}}$ mutant mice are more susceptible to the acute toxicity of 402 APAP than wild-type mice. Taken together, this study supports the notion that Nrf2-sMaf 403 specifically recognizes CsMBE sequences, which is necessary to the cytoprotective function. 


\section{Discussion}

406 Since CsMBE and MARE share common core sequence, it has been uncertain how many 407 target binding sites and target genes are shared by the Nrf2-sMaf heterodimers and Maf 408 homodimers. In this study, we wish to clarify this issue, and have generated a knock-in line 409 of mice expressing $\mathrm{Nrf}^{\mathrm{A} 502 \mathrm{Y}}$ mutant. As summarized in Figure 7, we first verified that 410 CsMBE is substantially different from MARE in vivo, despite of their similarity in terms of 411 DNA sequences. Of note, while Nrf2-sMaf prefers A/G nucleotide at 5'-flanking region of 412 the core sequence (CsMBE, left side), $\mathrm{Nrf2}^{\mathrm{A} 502 \mathrm{Y}}$-sMaf prefers $\mathrm{C}$ at that position (right side), 413 similar to the Maf homodimer-binding site (MARE), demonstrating that the sequence 414 recognition of $\mathrm{Nrf2}^{\mathrm{A} 502 \mathrm{Y}}$ shifts drastically from CsMBE to MARE in peritoneal macrophages 415 in vivo. The common binding sites of $\mathrm{Nrf2}$ and $\mathrm{Nrf} 2^{\mathrm{A} 502 \mathrm{Y}}$ do not show preference between $416 \mathrm{~A} / \mathrm{G} / \mathrm{C}$ nucleotides at the position. Of note, RNA-Seq data revealed that $\mathrm{Nrf} 2^{\mathrm{A} 502 \mathrm{Y}}$ 417 substantially lost the ability to support the expression of majority of the cytoprotective genes and, showing very good agreement with the results, $\mathrm{Nrf2}^{\mathrm{A} 502 \mathrm{Y}}$ mutant mice are severely 419 susceptible to the APAP toxicity. Based on these results, we conclude that the Nrf2-sMaf 420 heterodimers have acquired the CsMBE recognition during molecular evolution, and this 421 progress is critical for the cytoprotective functions of our body.

422 An ancestor of CNC family proteins is SKN-1 in Caenorhabditis elegans [25, 53, 54]. SKN-1 regulates a set of cytoprotective genes responding to oxidative stress as is the case 424 for vertebrate $\mathrm{Nrf} 2$ [53]. Of note, despite of the functional similarity to $\mathrm{Nrf} 2, \mathrm{SKN}-1$ 425 recognizes $\mathrm{ATGA}(\mathrm{G} / \mathrm{A})$ motif as a monomer. On the other hand, a CNC family protein in 426 Drosophila melanogaster, $\mathrm{CncC}$, forms a heterodimer with sMaf protein, Maf-S, and 427 together recognize $\operatorname{CsMBE}[(\mathrm{A} / \mathrm{G}) \mathrm{TGA}(\mathrm{G} / \mathrm{C}) \mathrm{nnnGC}]$ [23]. The SKN-1 binding motif is conserved within CsMBE (underlined), indicating the CNC transcription factors acquired ability to recognize extended cis-element by forming heterodimer with sMaf proteins in 
430 process of the molecular evolution. We surmise that the acquired long cis-acting element 431 enables vertebrates to execute the strict gene regulation in their huge genomes through 432 competing with the other transcription factors sharing partly the cis-element. Furthermore, 433 differences of CsMBE and MARE reduce mutual interference between CNC-sMaf 434 heterodimers and Maf homodimers, resulting in selective activation of the genes required in 435 the response against oxidative and xenobiotic stresses.

436 Several reports indicate the importance of TMA motif at 5' flanking region of CsMBE $437[47,48,50]$. Showing good agreement with the studies, we found that TMA, especially TCA, 438 motifs are enriched in WT-specific and Common sites but not in AY-specific sites. While 439 the TCA motif appears to play important roles in Nrf2-sMaf heterodimer binding to CsMBE, 440 it still remains unclear which factor recognizes the TCA motif and how the binding of Nrf2 441 to CsMBE is stabilized upon the presence of the TCA motif.

442 On the other hand, since $\mathrm{Nrf}^{\mathrm{A} 502 \mathrm{Y}}$-sMaf heterodimers recognize MARE, Nrf2 ${ }^{\mathrm{A} 502 \mathrm{Y}}$-sMaf 443 has the potential to affect MARE-dependent transcription of large Maf and sMaf 444 homodimers. Large Maf proteins (c-Maf, MafA/L-Maf, MafB and Nrl) play critical roles in 445 maintenance of homeostasis and ontogeny, including lens development, glucose homeostasis 446 and macrophage differentiation [53, 55-57]. Although accumulation of $\mathrm{Nrf} 2^{\mathrm{A} 502 \mathrm{Y}}$ may 447 interfere these biological effects, we did not observe obvious abnormality in the $N r f 2^{A Y / A Y}$ 448 mice except for high susceptibility to oxidative and xenobiotic stresses. These may be due to 449 two reasons. First, Keap1 constitutively degrades $\mathrm{Nrf2}$ and $\mathrm{Nrf}^{\mathrm{A} 502 \mathrm{Y}}$ under normal 450 conditions, so that without a challenge of chemical Nrf2-inducers $\operatorname{Nrf}^{\mathrm{A} 502 \mathrm{Y}}$ does not 451 accumulate massively in our body. The pharmacological induction of $\mathrm{Nrf2}$ and $\mathrm{Nrf} 2^{\mathrm{A} 502 \mathrm{Y}}$ 452 accumulation by DEM or other inducers is transient. Second, compared with the 453 pharmacological induction, genetic induction by knocking-out or knocking-down of Keap1 454 is potent and constitutive, and therefore results in adverse effects [58, 59]. We surmise that 
analyses using genetic induction of Nrf2 may provide clues about this issue.

In addition to WT-specific (CsMBE) and AY-specific (MARE) binding sites, we identified the Common binding sites that bind both Nrf2-sMaf and $\mathrm{Nrf2}^{\mathrm{A} 502 \mathrm{Y}}$-sMaf. Nevertheless, it still remains enigmatic why both Nrf2-sMaf and $\mathrm{Nrf}^{\mathrm{A} 502 \mathrm{Y}}$-sMaf heterodimers are able to bind to the Common sites. Our results show that the peaks belonging the Common binding sites are highly enrichment compared with both WT-specific and AY-specific binding sites, suggesting that the Common sites possess beneficial genomic conditions for binding of these factors, such as adjacent interacting motifs of other transcription factors that stabilize the binding of Nrf2-sMaf and sMaf homodimers.

We previously identified a competitive regulation between NF-E2 p45 and sMaf heterodimer (p45-sMaf) and sMaf homodimer in mouse megakaryocytes [60]. In the study, we identified that transgenic overexpression of sMaf severely repressed the p45-sMaf target gene expression and resulting proplatelet formation. Our present results further support the notion that binding of Nrf2-sMaf heterodimer to the Common genes seems to be competed with the sMaf homodimer and vise-versa, and the competition between Nrf2-sMaf heterodimer and sMaf homodimer affects expression profile of the Common genes. On the other hand, WT-specific genes seem to be regulated by the Nrf2-sMaf heterodimer without the competition.

In summary, we conclude that this study provides fundamental information that enlightens the elaborate transcriptional regulation of a subset of cytoprotective genes. The Nrf2-sMaf heterodimer sustains expression of the genes that are critical for cytoprotection against oxidative and xenobiotic stresses. In order to achieve a quick response against these stresses, it is crucial to select the target genes properly and timely, and CsMBE ensures the Nrf2-sMaf heterodimer to specify the proper genes without the interference or competition with large Maf and sMaf homodimers. Meanwhile, heterodimers of sMaf and CNC family 
480 transcription factors including Nrf1, Nrf2, Nrf3 and NF-E2 p45 regulates different target 481 genes via CsMBE recognition, indicating that the specificity of their gene regulation also 482 exists on different level (e.g. co-activator/co-repressor selection or epigenetic regulation) in 483 addition to simple recognition of DNA sequences [61]. Further analyses are necessary to 484 elucidate mechanisms how these transcription factors select specific target genes and exert 485 their diverse biological functions.

486 
487 Acknowledgments

488 We thank Ms. Eriko Naganuma and the Biomedical Research Core of Tohoku University 489 Graduate School of Medicine for technical support. This work was supported in part by 490 MEXT/JSPS KAKENHI $(24249015,26111002$ and 15 H02507 to M.Y., and 26461395 to 491 M.S.), AMED-CREST (to M.Y.), MEXT [a research program of the Project for 492 Development of Innovative Research on Cancer Therapeutics (P-Direct)], the Naito 493 Foundation, the Mitsubishi Foundation, and the Takeda Science Foundation (to M.Y.). A.O. 494 was a JSPS Research Fellow.

495 


\section{REFERENCES}

497 [1] Itoh, K.; Chiba, T.; Takahashi, S.; Ishii, T.; Igarashi, K.; Katoh, Y.; Oyake, T.; 498 Hayashi, N.; Satoh, K.; Hatayama, I.; Yamamoto, M.; Nabeshima, Y. An Nrf2/small Maf 499 heterodimer mediates the induction of phase II detoxifying enzyme genes through 500 antioxidant response elements. Biochem Biophys Res Commun 236:313-322; 1997.

501 [2] Taguchi, K.; Motohashi, H.; Yamamoto, M. Molecular mechanisms of the 502 Keap1-Nrf2 pathway in stress response and cancer evolution. Genes Cells 16:123-140; 5032011.

504 [3] Motohashi, H.; Katsuoka, F.; Engel, J. D.; Yamamoto, M. Small Maf proteins 505 serve as transcriptional cofactors for keratinocyte differentiation in the Keap1-Nrf2 506 regulatory pathway. Proc Natl Acad Sci U S A 101:6379-6384; 2004.

507 [4] Itoh, K.; Wakabayashi, N.; Katoh, Y.; Ishii, T.; Igarashi, K.; Engel, J. D.; 508 Yamamoto, M. Keap1 represses nuclear activation of antioxidant responsive elements by 509 Nrf2 through binding to the amino-terminal Neh2 domain. Genes Dev 13:76-86; 1999.

510 [5] Kobayashi, A.; Kang, M. I.; Okawa, H.; Ohtsuji, M.; Zenke, Y.; Chiba, T.; 511 Igarashi, K.; Yamamoto, M. Oxidative stress sensor Keap1 functions as an adaptor for 512 Cul3-based E3 ligase to regulate proteasomal degradation of Nrf2. Mol Cell Biol 513 24:7130-7139; 2004.

514 [6] Igarashi, K.; Kataoka, K.; Itoh, K.; Hayashi, N.; Nishizawa, M.; Yamamoto, M. 515 Regulation of transcription by dimerization of erythroid factor NF-E2 p45 with small Maf 516 proteins. Nature 367:568-572; 1994.

517 [7] Itoh, K.; Igarashi, K.; Hayashi, N.; Nishizawa, M.; Yamamoto, M. Cloning and 518 characterization of a novel erythroid cell-derived CNC family transcription factor 519 heterodimerizing with the small Maf family proteins. Mol Cell Biol 15:4184-4193; 1995.

520 [8] Johnsen, O.; Skammelsrud, N.; Luna, L.; Nishizawa, M.; Prydz, H.; Kolstø, A. B. 521 Small Maf proteins interact with the human transcription factor TCF11/Nrf1/LCR-F1. $522 \quad$ Nucleic Acids Res 24:4289-4297; 1996.

523 [9] Oyake, T.; Itoh, K.; Motohashi, H.; Hayashi, N.; Hoshino, H.; Nishizawa, M.; 524 Yamamoto, M.; Igarashi, K. Bach proteins belong to a novel family of BTB-basic leucine 525 zipper transcription factors that interact with MafK and regulate transcription through the $526 \quad$ NF-E2 site. Mol Cell Biol 16:6083-6095; 1996.

527 [10] Rushmore, T. H.; Morton, M. R.; Pickett, C. B. The antioxidant responsive 528 element. Activation by oxidative stress and identification of the DNA consensus sequence 529 required for functional activity. J Biol Chem 266:11632-11639; 1991.

530 [11] Hirotsu, Y.; Katsuoka, F.; Funayama, R.; Nagashima, T.; Nishida, Y.; Nakayama, 531 K.; Engel, J. D.; Yamamoto, M. Nrf2-MafG heterodimers contribute globally to antioxidant 532 and metabolic networks. Nucleic Acids Res 40:10228-10239; 2012.

533 [12] Friling, R. S.; Bensimon, A.; Tichauer, Y.; Daniel, V. Xenobiotic-inducible 534 expression of murine glutathione S-transferase Ya subunit gene is controlled by an electrophile-responsive element. Proc Natl Acad Sci U S A 87:6258-6262; 1990.

[13] Mignotte, V.; Eleouet, J. F.; Raich, N.; Romeo, P. H. Cis- and trans-acting elements involved in the regulation of the erythroid promoter of the human porphobilinogen deaminase gene. Proc Natl Acad Sci U S A 86:6548-6552; 1989.

541 Biol 14:700-712; 1994.

542 [15] Kataoka, K.; Fujiwara, K. T.; Noda, M.; Nishizawa, M. MafB, a new Maf family

543 transcription activator that can associate with Maf and Fos but not with Jun. Mol Cell Biol 
[16] Kerppola, T. K.; Curran, T. A conserved region adjacent to the basic domain is required for recognition of an extended DNA binding site by $\mathrm{Maf} / \mathrm{Nrl}$ family proteins. Oncogene 9:3149-3158; 1994.

[17] Kataoka, K.; Noda, M.; Nishizawa, M. Maf nuclear oncoprotein recognizes sequences related to an AP-1 site and forms heterodimers with both Fos and Jun. Mol Cell Biol 14:700-712; 1994.

[18] Yamamoto, T.; Kyo, M.; Kamiya, T.; Tanaka, T.; Engel, J. D.; Motohashi, H.; Yamamoto, M. Predictive base substitution rules that determine the binding and transcriptional specificity of Maf recognition elements. Genes Cells 11:575-591; 2006.

[19] Kurokawa, H.; Motohashi, H.; Sueno, S.; Kimura, M.; Takagawa, H.; Kanno, Y.; Yamamoto, M.; Tanaka, T. Structural basis of alternative DNA recognition by Maf transcription factors. Mol Cell Biol 29:6232-6244; 2009.

[20] Kusunoki, H.; Motohashi, H.; Katsuoka, F.; Morohashi, A.; Yamamoto, M.; Tanaka, T. Solution structure of the DNA-binding domain of MafG. Nat Struct Biol 9:252-256; 2002.

[21] Consortium, C. e. S. Genome sequence of the nematode C. elegans: a platform for investigating biology. Science 282:2012-2018; 1998.

[22] Li, M. A.; Alls, J. D.; Avancini, R. M.; Koo, K.; Godt, D. The large Maf factor Traffic Jam controls gonad morphogenesis in Drosophila. Nat Cell Biol 5:994-1000; 2003.

[23] Rahman, M. M.; Sykiotis, G. P.; Nishimura, M.; Bodmer, R.; Bohmann, D. Declining signal dependence of Nrf2-MafS-regulated gene expression correlates with aging phenotypes. Aging Cell 12:554-562; 2013.

[24] Takagi, Y.; Kobayashi, M.; Li, L.; Suzuki, T.; Nishikawa, K.; Yamamoto, M. MafT, a new member of the small Maf protein family in zebrafish. Biochem Biophys Res Commun 320:62-69; 2004.

[25] Blackwell, T. K.; Bowerman, B.; Priess, J. R.; Weintraub, H. Formation of a monomeric DNA binding domain by Skn-1 bZIP and homeodomain elements. Science 266:621-628; 1994.

[26] McGinnis, N.; Ragnhildstveit, E.; Veraksa, A.; McGinnis, W. A cap 'n' collar protein isoform contains a selective Hox repressor function. Development 125:4553-4564; 1998.

[27] Kobayashi, M.; Itoh, K.; Suzuki, T.; Osanai, H.; Nishikawa, K.; Katoh, Y.; Takagi, Y.; Yamamoto, M. Identification of the interactive interface and phylogenic conservation of the Nrf2-Keap1 system. Genes Cells 7:807-820; 2002.

[28] Timme-Laragy, A. R.; Karchner, S. I.; Franks, D. G.; Jenny, M. J.; Harbeitner, R. C.; Goldstone, J. V.; McArthur, A. G.; Hahn, M. E. Nrf2b, novel zebrafish paralog of oxidant-responsive transcription factor NF-E2-related factor 2 (NRF2). J Biol Chem 287:4609-4627; 2012.

[29] Kimura, M.; Yamamoto, T.; Zhang, J.; Itoh, K.; Kyo, M.; Kamiya, T.; Aburatani, H.; Katsuoka, F.; Kurokawa, H.; Tanaka, T.; Motohashi, H.; Yamamoto, M. Molecular basis distinguishing the DNA binding profile of Nrf2-Maf heterodimer from that of Maf homodimer. J Biol Chem 282:33681-33690; 2007.

[30] Dlakić, M.; Grinberg, A. V.; Leonard, D. A.; Kerppola, T. K. DNA sequence-dependent folding determines the divergence in binding specificities between Maf and other bZIP proteins. EMBO J 20:828-840; 2001.

[31] Mashiko, D.; Fujihara, Y.; Satouh, Y.; Miyata, H.; Isotani, A.; Ikawa, M. Generation of mutant mice by pronuclear injection of circular plasmid expressing Cas9 and single guided RNA. Sci Rep 3:3355; 2013. 

Jiang, W.; Marraffini, L. A.; Zhang, F. Multiplex genome engineering using CRISPR/Cas systems. Science 339:819-823; 2013.

596 [33] Ishii, T.; Itoh, K.; Takahashi, S.; Sato, H.; Yanagawa, T.; Katoh, Y.; Bannai, S.; Yamamoto, M. Transcription factor Nrf2 coordinately regulates a group of oxidative stress-inducible genes in macrophages. J Biol Chem 275:16023-16029; 2000.

599 [34] Maruyama, A.; Tsukamoto, S.; Nishikawa, K.; Yoshida, A.; Harada, N.; Motojima,

600 K.; Ishii, T.; Nakane, A.; Yamamoto, M.; Itoh, K. Nrf2 regulates the alternative first exons 601 of CD36 in macrophages through specific antioxidant response elements. Arch Biochem 602 Biophys 477:139-145; 2008.

603 [35] Katsuoka, F.; Yokozawa, J.; Tsuda, K.; Ito, S.; Pan, X.; Nagasaki, M.; Yasuda, J.; 604 Yamamoto, M. An efficient quantitation method of next-generation sequencing libraries by using MiSeq sequencer. Anal Biochem 466:27-29; 2014.

Methods 9:357-359; 2012.

608 [37] Thorvaldsdóttir, H.; Robinson, J. T.; Mesirov, J. P. Integrative Genomics Viewer 609 (IGV): high-performance genomics data visualization and exploration. Brief Bioinform $610 \quad \mathbf{1 4 : 1 7 8 - 1 9 2 ; 2 0 1 3 .}$

611 [38] Zhang, Y.; Liu, T.; Meyer, C. A.; Eeckhoute, J.; Johnson, D. S.; Bernstein, B. E.; 612 Nusbaum, C.; Myers, R. M.; Brown, M.; Li, W.; Liu, X. S. Model-based analysis of 613 ChIP-Seq (MACS). Genome Biol 9:R137; 2008.

614 [39] Machanick, P.; Bailey, T. L. MEME-ChIP: motif analysis of large DNA datasets. Bioinformatics 27:1696-1697; 2011.

616 [40] Trapnell, C.; Pachter, L.; Salzberg, S. L. TopHat: discovering splice junctions with 617 RNA-Seq. Bioinformatics 25:1105-1111; 2009.

618 [41] Trapnell, C.; Williams, B. A.; Pertea, G.; Mortazavi, A.; Kwan, G.; van Baren, M. 619 J.; Salzberg, S. L.; Wold, B. J.; Pachter, L. Transcript assembly and quantification by 620 RNA-Seq reveals unannotated transcripts and isoform switching during cell differentiation. 621 Nat Biotechnol 28:511-515; 2010.

622 [42] Subramanian, A.; Tamayo, P.; Mootha, V. K.; Mukherjee, S.; Ebert, B. L.; Gillette, 623 M. A.; Paulovich, A.; Pomeroy, S. L.; Golub, T. R.; Lander, E. S.; Mesirov, J. P. Gene set 624 enrichment analysis: a knowledge-based approach for interpreting genome-wide expression profiles. Proc Natl Acad Sci U S A 102:15545-15550; 2005. [43] Hayes, J. D.; Dinkova-Kostova, A. T. The Nrf2 regulatory network provides an interface between redox and intermediary metabolism. Trends Biochem Sci 39:199-218; 2014.

629 [44] Edgar, R.; Domrachev, M.; Lash, A. E. Gene Expression Omnibus: NCBI gene 630 expression and hybridization array data repository. Nucleic Acids Res 30:207-210; 2002.

631 [45] Higgins, L. G.; Kelleher, M. O.; Eggleston, I. M.; Itoh, K.; Yamamoto, M.; Hayes, 632 J. D. Transcription factor Nrf2 mediates an adaptive response to sulforaphane that protects 633 fibroblasts in vitro against the cytotoxic effects of electrophiles, peroxides and redox-cycling 634 agents. Toxicol Appl Pharmacol 237:267-280; 2009.

635 [46] Yang, Y.; Fear, J.; Hu, J.; Haecker, I.; Zhou, L.; Renne, R.; Bloom, D.; McIntyre, 636 L. M. Leveraging biological replicates to improve analysis in ChIP-seq experiments. Comput 637 Struct Biotechnol J 9:e201401002; 2014.

638 [47] Nioi, P.; McMahon, M.; Itoh, K.; Yamamoto, M.; Hayes, J. D. Identification of a 639 novel Nrf2-regulated antioxidant response element (ARE) in the mouse NAD(P)H:quinone 640 oxidoreductase 1 gene: reassessment of the ARE consensus sequence. Biochem $J$ $641 \quad 374: 337-348 ; 2003$. 

Natl Acad Sci U S A 94:5361-5366; 1997.

644 [49] Katsuoka, F.; Motohashi, H.; Engel, J. D.; Yamamoto, M. Nrf2 transcriptionally activates the mafG gene through an antioxidant response element. $J$ Biol Chem 280:4483-4490; 2005. element. Identification of the nucleotide sequence required for basal and inducible activity and detection of antioxidant response element-binding proteins in hepatoma and non-hepatoma cell lines. J Biol Chem 270:24468-24474; 1995.

651 [51] Itoh, K.; Mochizuki, M.; Ishii, Y.; Ishii, T.; Shibata, T.; Kawamoto, Y.; Kelly, V.; 652 Sekizawa, K.; Uchida, K.; Yamamoto, M. Transcription factor Nrf2 regulates inflammation by mediating the effect of 15-deoxy-Delta(12,14)-prostaglandin j(2). Mol Cell Biol 24:36-45; 2004.

655 [52] Kobayashi, E.; Suzuki, T.; Yamamoto, M. Roles nrf2 plays in myeloid cells and related disorders. Oxid Med Cell Longev 2013:529219; 2013.

657 [53] An, J. H.; Blackwell, T. K. SKN-1 links C. elegans mesendodermal specification to a conserved oxidative stress response. Genes Dev 17:1882-1893; 2003.

659 [54] Carroll, A. S.; Gilbert, D. E.; Liu, X.; Cheung, J. W.; Michnowicz, J. E.; Wagner, 660 G.; Ellenberger, T. E.; Blackwell, T. K. SKN-1 domain folding and basic region monomer 661 stabilization upon DNA binding. Genes Dev 11:2227-2238; 1997.

662 [55] Takeuchi, T.; Kudo, T.; Ogata, K.; Hamada, M.; Nakamura, M.; Kito, K.; Abe, Y.; 663 Ueda, N.; Yamamoto, M.; Engel, J. D.; Takahashi, S. Neither MafA/L-Maf nor MafB is essential for lens development in mice. Genes Cells 14:941-947; 2009.

666

667

668 Oishi, H.; Hamada, M.; Morito, N.; Hasegawa, K.; Kudo, T.; Engel, J. D.; Yamamoto, M.; Takahashi, S. MafA is a key regulator of glucose-stimulated insulin secretion. Mol Cell Biol 25:4969-4976; 2005.

670 R.; Moriguchi, T.; Kudo, T.; Takahashi, S. c-Maf is essential for the F4/80 expression in 671 macrophages in vivo. Gene 445:66-72; 2009.

672 [58] Wakabayashi, N.; Itoh, K.; Wakabayashi, J.; Motohashi, H.; Noda, S.; Takahashi, 673 S.; Imakado, S.; Kotsuji, T.; Otsuka, F.; Roop, D. R.; Harada, T.; Engel, J. D.; Yamamoto, 674 M. Keap1-null mutation leads to postnatal lethality due to constitutive Nrf2 activation. Nat Genet 35:238-245; 2003.

[59] Taguchi, K.; Maher, J. M.; Suzuki, T.; Kawatani, Y.; Motohashi, H.; Yamamoto, M. Genetic analysis of cytoprotective functions supported by graded expression of Keap1. Mol Cell Biol 30:3016-3026; 2010.

[60] Motohashi, H.; Katsuoka, F.; Shavit, J. A.; Engel, J. D.; Yamamoto, M. Positive or negative MARE-dependent transcriptional regulation is determined by the abundance of small Maf proteins. Cell 103:865-875; 2000.

686 
689 Figure 1. Generation of $\mathbf{N r f 2}^{\mathbf{A 5 0 2 Y}}$ knock-in mice. (A) Cis-element recognition by

690 Nrf2-sMaf heterodimers and Maf homodimers. Nrf2-sMaf heterodimer recognizes CsMBE,

691 while Maf homodimer recognizes MARE. An amino acid substitution of Nrf2 502nd alanine

692 to tyrosine is expected to change the recognition specificity of Nrf2-sMaf heterodimers from

693 CsMBE to MARE. Critical GC sequence of MARE in the 5' of TRE and corresponding A/G

694 sequence of CsMBE are underlined, and described as lock-and-key models in the scheme.

695 (B) Domain structure of Nrf2 protein. The $502^{\text {nd }}$ residue of alanine (A, blue letter) positioned

696 at Nrf2-ECH homology 1 (Neh1) domain is replaced by tyrosine (Y, red letter). (C)

697 Cas9/guide RNA (gRNA)-targeting site in Nrf2 gene. The sequences of gRNA and the donor

698 DNA co-injected for targeting mutagenesis are underlined. The proto spacer adjacent motif

699 (PAM) sequence is indicated by green. The $502^{\text {nd }}$ alanine residue (blue) and corresponding

700 tyrosine residue (red) are shown. (D) Representative sequences of Nrf2 targeting region of

701 genomic DNA from $N r f 2^{+/+}, N r f 2^{\mathrm{AY} /+}$, and $\mathrm{Nrf} 2^{\mathrm{AY} / \mathrm{AY}}$ mice. The codons encoding alanine

702 (GCC) and tyrosine (TAC) are underlined. (E) Representative sequences of Nrf2 targeting 703 region of cDNA in $N r f 2^{\mathrm{AY} /+}$ mice. (F) The growth curves of $N r f 2^{+/+}(+/+$, blue circle, male $704 \mathrm{n}=5$, female $\mathrm{n}=8$ ) and $N r 2^{\mathrm{AY} / \mathrm{AY}}$ (AY/AY, pink square, male $\mathrm{n}=6$, female $\mathrm{n}=6$ ) male and 705 female mice. Data represent the mean \pm standard deviations (S.D.).

707 Figure 2. $\mathrm{Nrf} 2^{A \mathrm{Y} / A Y}$ macrophages are susceptible to cytotoxic effects of xenobiotics. (A)

708 Scheme for induction of peritoneal macrophages and Nrf2 accumulation. (B) Cellular 709 surface marker profiles of $\mathrm{Nrf}^{+/+}$and $\mathrm{Nrf} 2^{\mathrm{AY} / \mathrm{AY}}$ macrophages. The cells recovered from $710 \mathrm{Nrf}^{+/+}$and $\mathrm{Nrf} 2^{\mathrm{AY} / \mathrm{AY}}$ mice were stained with Mac1, Gr-1, and F4/80 antibodies. (C) Nrf2 711 mRNA levels in the $N r f 2^{+/+}$and $N r f 2^{\mathrm{AY} / \mathrm{AY}}$ macrophages under basal (white) and 712 DEM-treated (gray) states. The abundance of each mRNA was normalized to rRNA. 
713 Average values for $\mathrm{Nrf}^{+/+}$macrophages under basal state were set to $1 . \mathrm{Nrf2^{+/+ }} \mathrm{n}=4$,

$714 N r f 2^{A Y / A Y} \mathrm{n}=6$. (D and E) Protein levels in the $N r f 2^{+/+}$and $N r f 2^{\mathrm{AY} / \mathrm{AY}}$ macrophages under basal

715 (white) and DEM-treated (gray) states. Average values for $\mathrm{Nrf} 2^{+/+}$macrophages under basal

716 state were set to 1. Lamin B was used as a loading control. $N r f 2^{+/+} \mathrm{n}=6, N r f 2^{A Y / A Y} \mathrm{n}=5$. (F)

717 Relative viabilities of $N r f 2^{+/+}(\mathrm{WT}$, blue circle, $\mathrm{n}=5)$ and $N r f 2^{\mathrm{AY} / \mathrm{AY}}$ (AY, red square, $\mathrm{n}=8$ )

718 macrophages under menadione treatment. Note that $N r f 2^{\mathrm{AY} / \mathrm{AY}}$ macrophages are prone to die

719 upon the menadione treatment. Graph data represent the mean \pm SD. Student's $t$-test

720 (two-tailed), ${ }^{*} P<0.05$, n.s., not significant.

Figure 3. A502Y mutation of Nrf2 converts the binding preference of Nrf2 in vivo. (A)

723 Venn diagram showing the overlap between the Nrf2 (blue circle) and $\mathrm{Nrf2}^{\mathrm{A} 502 \mathrm{Y}}$ (red circle)

724 binding sites. The numbers in parenthesis show total numbers of $\mathrm{Nrf2}$ or $\mathrm{Nrf}^{\mathrm{A} 502 \mathrm{Y}}$ binding 725 sites. (B) Representative binding peaks of $\mathrm{Nrf2}$ and $\mathrm{Nrf}^{\mathrm{A} 502 \mathrm{Y}}$. The representative histograms 726 of tag count observed in ChIP-ed and Input samples from WT-specific, Common, and 727 AY-specific sites are shown. Scale bars, 1 kb. (C) Fold-enrichment (ChIP-ed/Input) values 728 of Nrf2 (left panel) and $\mathrm{Nrf2}^{\mathrm{A} 502 \mathrm{Y}}$ (right panel) binding at WT-specific (WT-sp.), Common 729 (Comm.), and AY-specific (AY-sp.) sites. In the box plots, bottom and top of the boxes 730 correspond to the 25 th and 75 th percentiles and the internal band is the median. The bars 731 outside the boxes indicate the highest and lowest data within 1.5 interquartile ranges of the 732 upper and lower quartiles, respectively. Wilcoxon rank sum test, ${ }^{* * *} p<0.001$. (D) The 733 motifs enriched in Nrf2 and $\mathrm{Nrf}^{\mathrm{A} 502 \mathrm{Y}}$ binding sites. These motifs were identified using de 734 novo motif-discovery algorithm MEME-ChIP version 4.10.0. (E) Frequency of nucleotides 735 at position 0 in WT-specific (WT-sp.), Common (Comm.) and AY-specific (AY-sp.) sites. 736 Note that AY-sp. prefers C at this position, as is the case for MARE, while WT-sp. prefers 737 A/G at this position. (F) Frequency of TMA-containing CsMBE in WT-specific, Common, 
and AY-specific sites. The consensus sequence of the TMA-containing CsMBE is shown in top panel. The TMA motif (position -5 to $-3, \mathrm{M}=\mathrm{A}$ or $\mathrm{C}$ ) is indicated in red.

\section{Figure 4. Substitution of Nrf2 to $\mathrm{Nrf2}^{\mathrm{A502Y}}$ abrogates electrophilic stress response in}

mice. (A and B) Scatter plots comparing transcript levels at basal (x-axis) and DEM-induced

743 (y-axis) states in the $N r f 2^{+/+}\left(\mathrm{WT}\right.$, panel A) and $N r f 2^{\mathrm{AY} / \mathrm{AY}}$ (AY, panel B) macrophages. We

744 found that transcript levels of 696 and 706 genes were significantly upregulated and 745 downregulated by DEM $(q<0.05)$, respectively, in WT mouse macrophages. Similarly, 746 transcript levels of 149 and 254 genes were significantly upregulated and downregulated by 747 DEM $(q<0.05)$, respectively, in $N r 2^{\mathrm{AY} / \mathrm{AY}}$ mouse macrophages. These genes are plotted. 748 The numbers of upregulated (UP) and downregulated (DOWN) genes are shown at the upper 749 left and lower right, respectively. (C) Venn diagram showing the overlap between 750 differentially expressed genes (DEGs). DEGs in the $N r f 2^{+/+}$(WT) and $N r f 2^{\mathrm{AY} / \mathrm{AY}}$ (AY) 751 macrophages are shown in blue and red, respectively. The numbers in parenthesis show total 752 numbers of DEGs. Note that the genes responding to DEM, irrespective to the upregulated or 753 downregulated, are significantly reduced in $N r f 2^{\mathrm{AY} / \mathrm{AY}}$ macrophages. (D) Heat maps showing 754 relative expression levels of WT-specific (left), Common (middle) and AY-specific (right) 755 DEGs. The numbers of upregulated (UP) and downregulated (DOWN) genes are shown 756 above the heat maps. (E) KEGG biological pathways enriched in WT-specific DEGs.

Figure 5. Nrf2 ${ }^{\mathrm{A502Y}}$ mutant fails to induce Nrf2 target genes. (A) GSEA histogram of the 759 gene set containing well-known Nrf2 target genes. We compared a gene set that contains 760 Nrf2-target genes differentially expressed by DEM specifically in the $\mathrm{Nrf}^{+/+}$macrophages 761 but not in $\mathrm{Nrf}^{\mathrm{A} 502 \mathrm{Y}}$ macrophages with a known Nrf2-dependent gene set [11, 43]. The 762 enrichment score (ES) and the nominal $\mathrm{p}$ value are indicated. The gene expression spectrum 
763 (red to blue) is shown in the bottom of the histogram. Note that known Nrf2 target genes are 764 contained in the DEM-upregulated gene group in the $\mathrm{Nrf}^{+/+}$macrophages. (B and C)

765 Transcriptome analyses of representative genes induced by DEM. Typical Nrf2-target genes 766 that are induced specifically in $N r f 2^{+/+}(\mathrm{WT}, \mathrm{n}=3)$ macrophages, but not in the $N r f 2^{\mathrm{AY} / \mathrm{AY}}$ 767 (AY, n=3) macrophages, as shown in panel B. Note that there exist a group of genes that are 768 induced by DEM both in the $N r f 2^{+/+}$and $N r f 2^{\mathrm{AY} / \mathrm{AY}}$ macrophages, as shown in panel C. 769 Expression levels of the genes are expressed as FPKM (fragments per kilobase of exon per 770 million fragments). Graph data represent the mean $\pm \mathrm{SD}$. Paired t-test, ${ }^{*} p<0.05$. (D and E) 771 Representative histograms of $\mathrm{Nrf} 2$ and $\mathrm{Nrf}^{\mathrm{A} 502 \mathrm{Y}}$ occupancy in proximal region of 772 representative Nrf2 target genes. WT-specific binding and common binding are shown in 773 panels D and E, respectively.

Figure 6. Nrf2 $^{\mathrm{A502Y}}$ mice are sensitive to APAP-induced liver injury. (A) Scheme for the analysis of sensitivity of $\mathrm{Nrf}^{\mathrm{A} 502 \mathrm{Y}}$ mice to acetaminophen (APAP) toxicity. (B) AST (left)

777 and ALT (right) levels in the plasma of $\mathrm{Nrf}^{+/+}\left(\mathrm{WT}\right.$, blue circle) and $N r f 2^{\mathrm{AY} / \mathrm{AY}}$ (AY, red 778 square) mice. The plots and bars show individual values and means, respectively. Student's $t$-test (two-tailed), ${ }^{*} p<0.05$. (C) Liver pathology of high-dose APAP-treated $\mathrm{Nrf}^{+/+}$and $N r f 2^{\mathrm{AY} / \mathrm{AY}}$ mice. HE staining of representative liver sections of $N r f 2^{+/+}(\mathrm{WT})$ and $N r f 2^{\mathrm{AY} / \mathrm{AY}}$

781 (AY) mice are shown. Scale bars, $100 \mu \mathrm{m}$.

783 Figure 7. Schematic diagram of target recognition by Nrf2 and Nrf2 ${ }^{\mathbf{A 5 0 2 Y}}$. Nrf2-sMaf prefers CsMBE harboring an $\mathrm{A} / \mathrm{G}$ nucleotide at the 5'-flanking region of the core sequence 785 (left side), while $\mathrm{Nrf}^{\mathrm{A} 502 \mathrm{Y}}$-sMaf prefers MARE harboring a $\mathrm{C}$ nucleotide at that position 786 (right side), similar to sMaf homodimer. The common binding sites of $\mathrm{Nrf} 2$ and $\mathrm{Nrf} 2^{\mathrm{A} 502 \mathrm{Y}}$ do 787 not show preference between $\mathrm{A} / \mathrm{G} / \mathrm{C}$ nucleotides at that position (middle). Majority of the 
788 cytoprotective genes (e.g., detoxifying and antioxidant genes) are regulated by Nrf2 in a 789 CsMBE-dependent manner. $\mathrm{Nrf}^{\mathrm{A} 502 \mathrm{Y}}$ fails to recognize CsMBE and therefore fails to 790 induce cytoprotective genes, which results in a weak defense in the $N r f 2^{\mathrm{AY} / \mathrm{AY}}$ mice. 

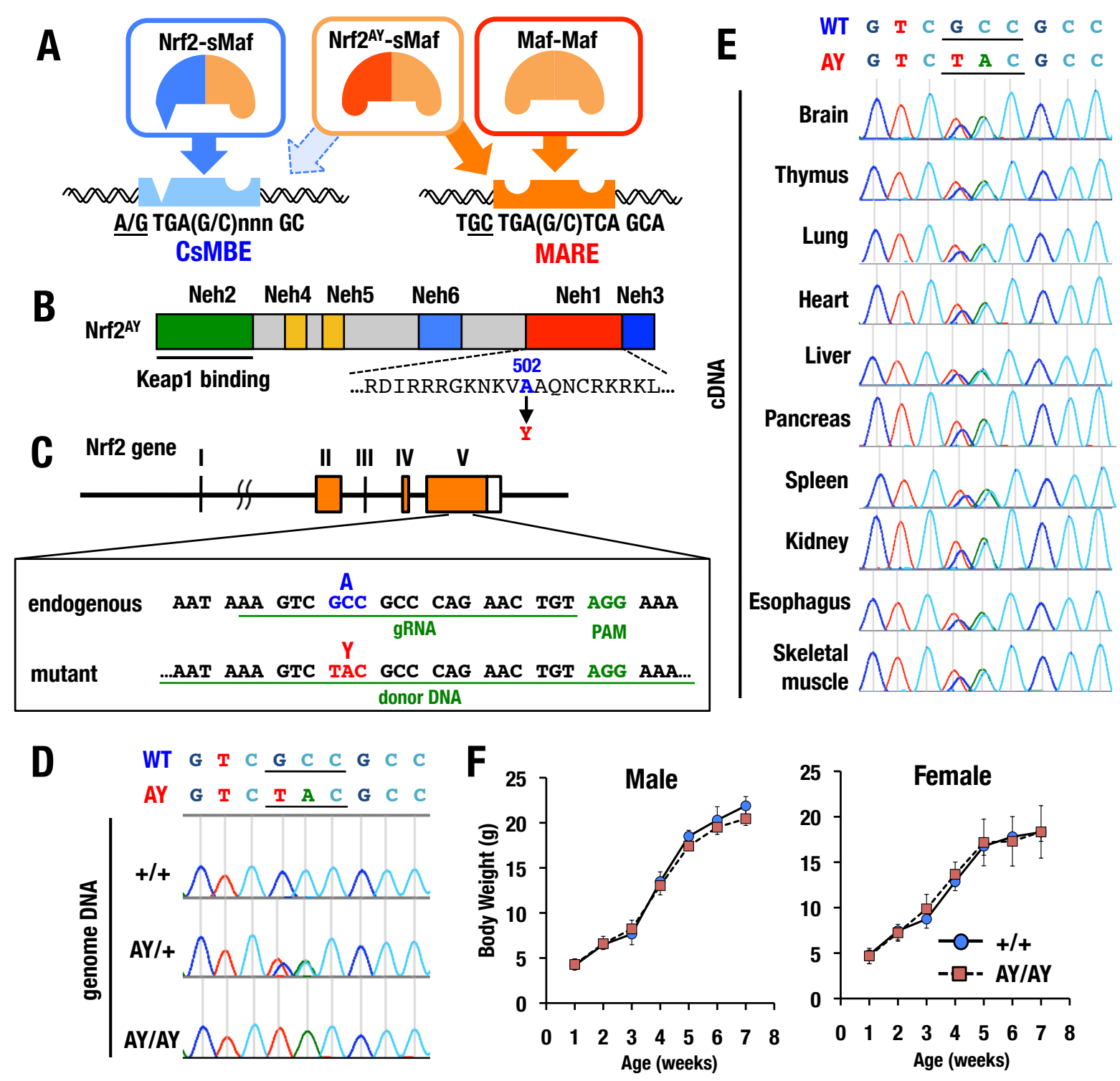

Figure 1 
A
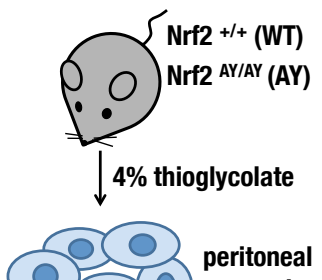

(1) macrophages

$$
\downarrow \text { DEM }
$$

Analysis

C Nrf2 (mRNA)

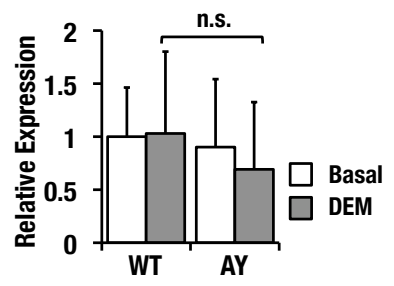

E Nrf2 (Protein)

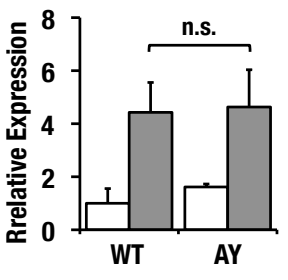

B
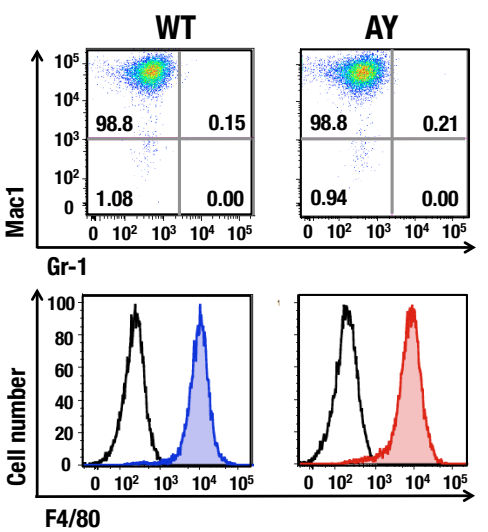

D

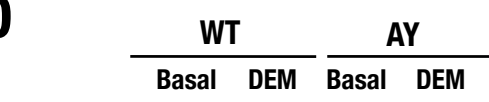

Lamin B

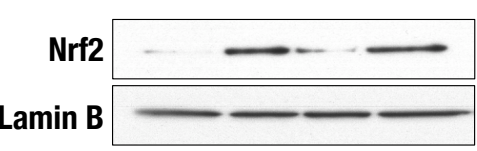

F $1.4 \quad-0-$ WT $\quad-\square-A Y$

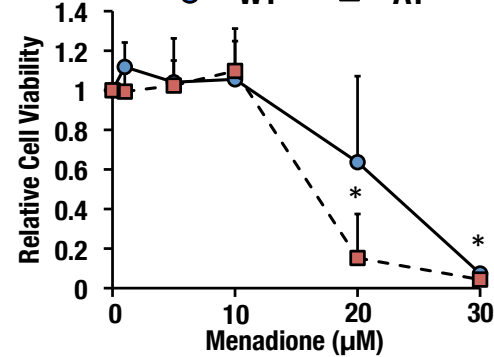

Figure 2 

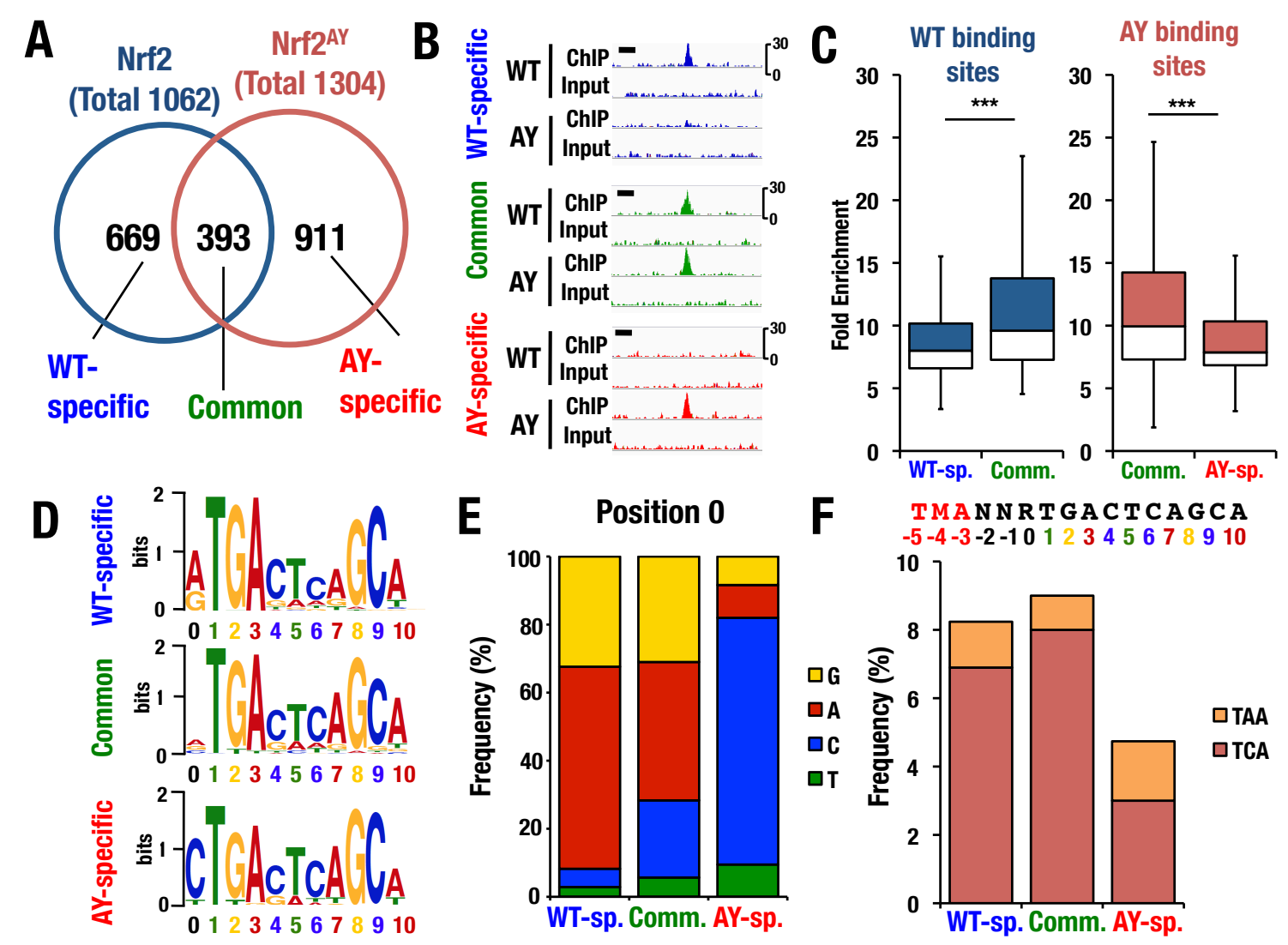

Figure 3 

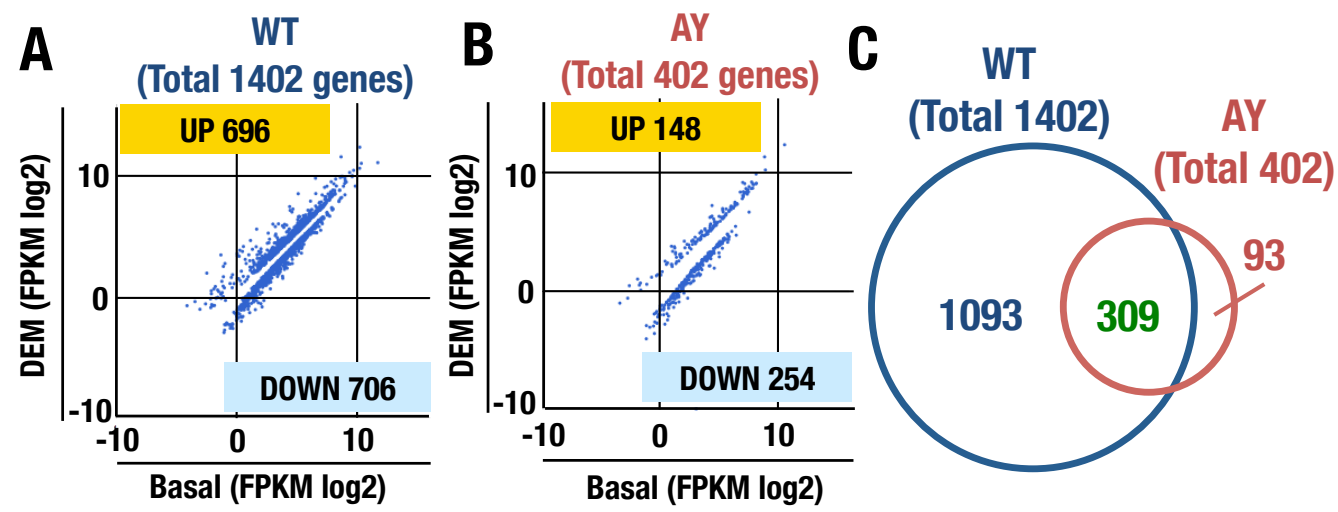

D WT-specific DEGs (UP: 586, DOWN: 507)

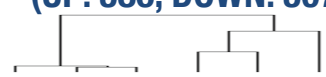

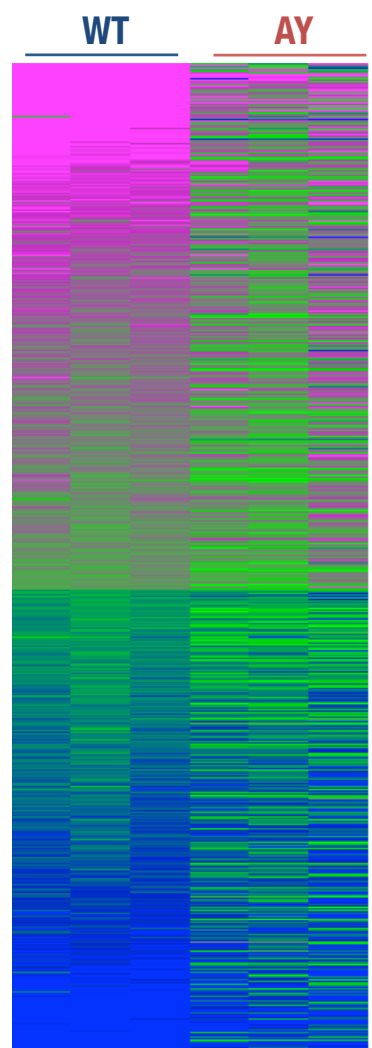

Common DEGs

AY-specific DEGs

(UP: 110, DOWN: 199)

(UP: 38, DOWN: 55)
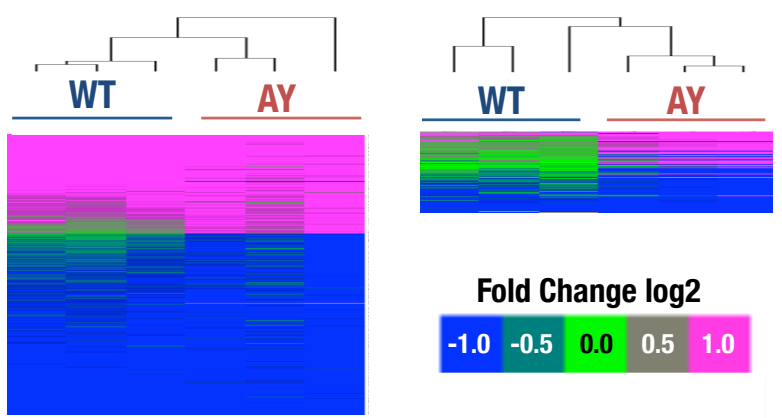

Fold Change log2

$\begin{array}{llllll}-1.0 & -0.5 & 0.0 & 0.5 & 1.0\end{array}$

E KEGG pathway analysis on WT-specific DEGs

\begin{tabular}{lc}
\hline \multicolumn{1}{c}{ Term } & $\begin{array}{c}\text { corrected } \\
\text { p-value }\end{array}$ \\
\hline Upregulated genes & \\
\hline Proteasome & $6.85 \times 10^{-4}$ \\
Glutathione metabolism & $5.90 \times 10^{-3}$ \\
Pentose phosphate pathway & 0.012 \\
Amyotrophic lateral sclerosis (ALS) & 0.032 \\
Apoptosis & 0.030 \\
\hline Downregulated genes & \\
\hline Fc gamma R-mediated phagocytosis & $7.72 \times 10^{-3}$ \\
Regulation of actin cytoskeleton & $4.65 \times 10^{-3}$ \\
Chemokine signaling pathway & 0.016 \\
Jak-STAT signaling pathway & 0.025 \\
Focal adhesion & 0.023 \\
Leukocyte transendothelial migration & 0.028 \\
\hline
\end{tabular}



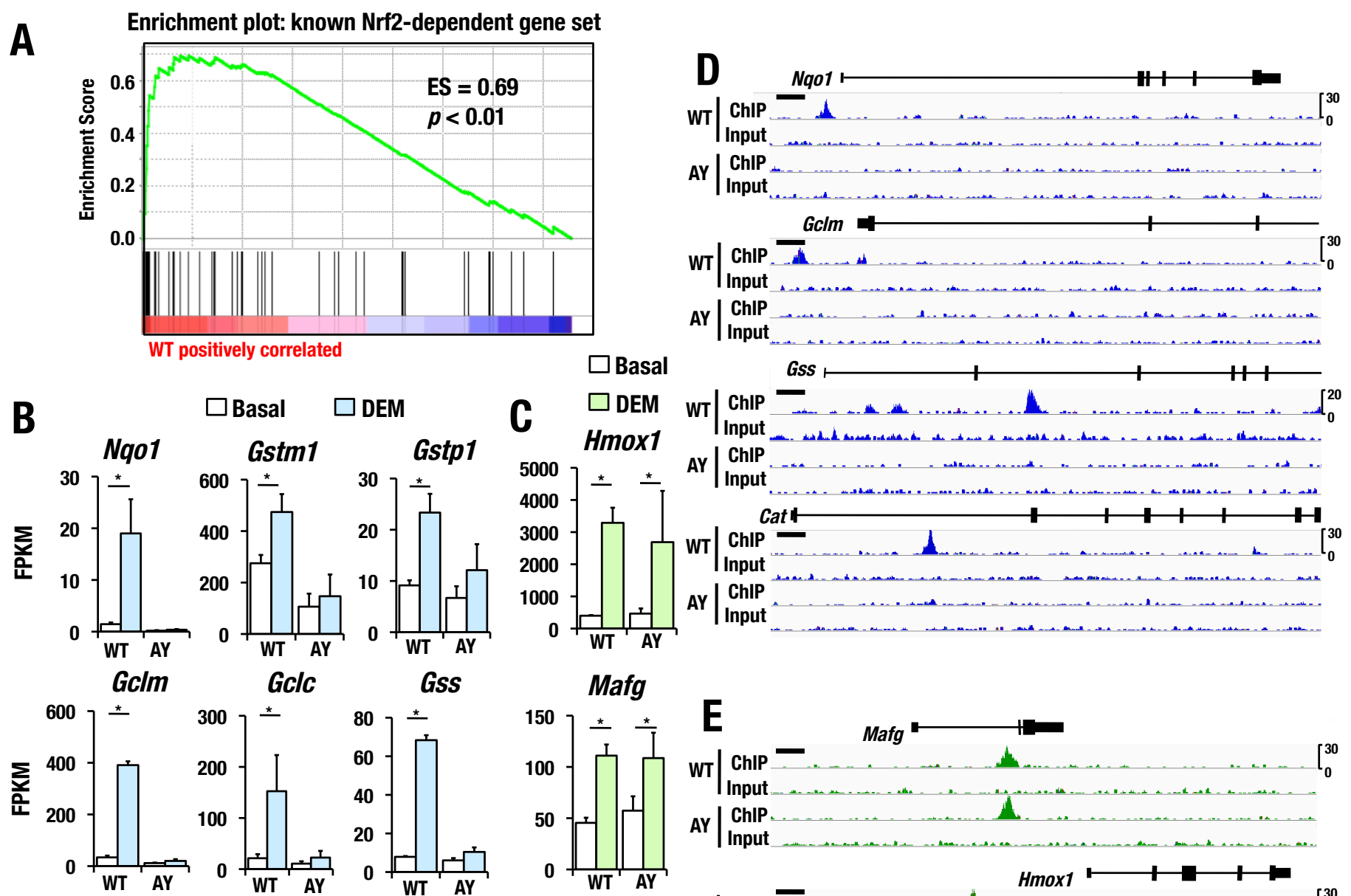

AY $\left.\right|_{\text {Input }} ^{\text {ChP }}$
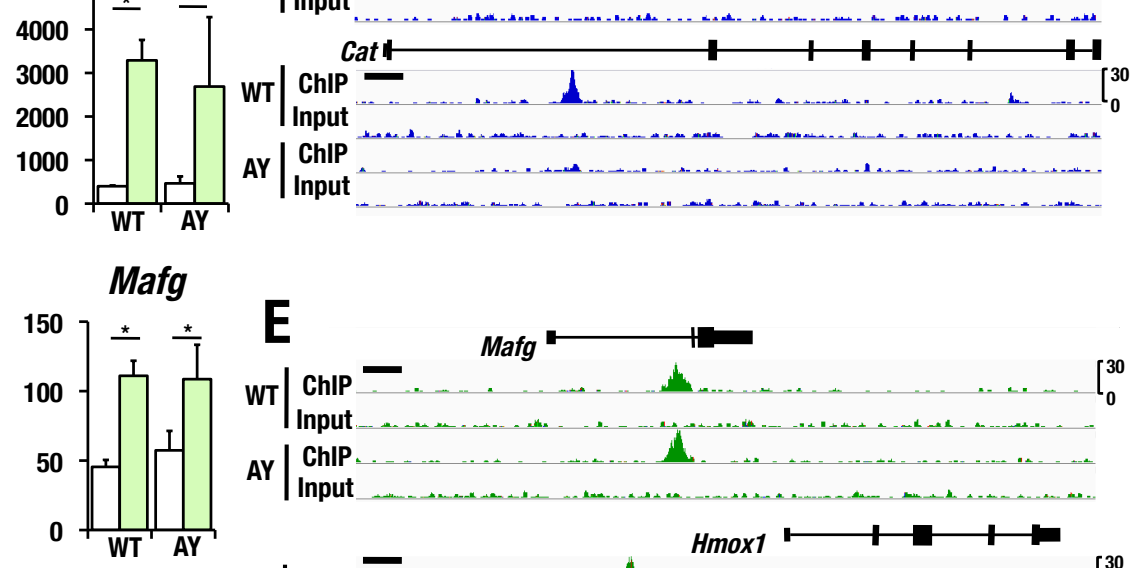

E
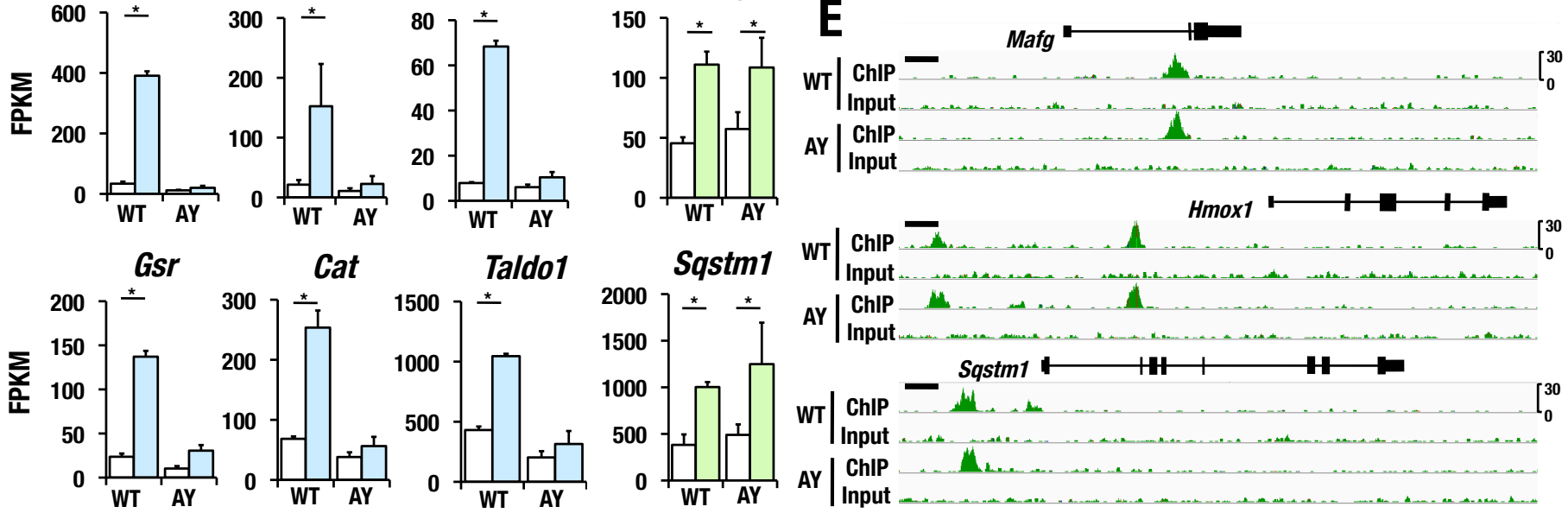

Ar $\mid$ ChP _...........

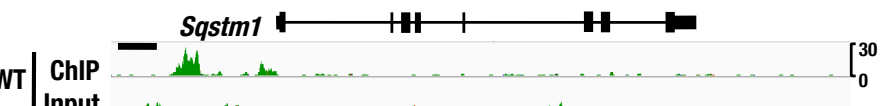

AY $\mid \begin{gathered}\text { ChP } \\ \text { Input }\end{gathered}$

\section{Figure 5}




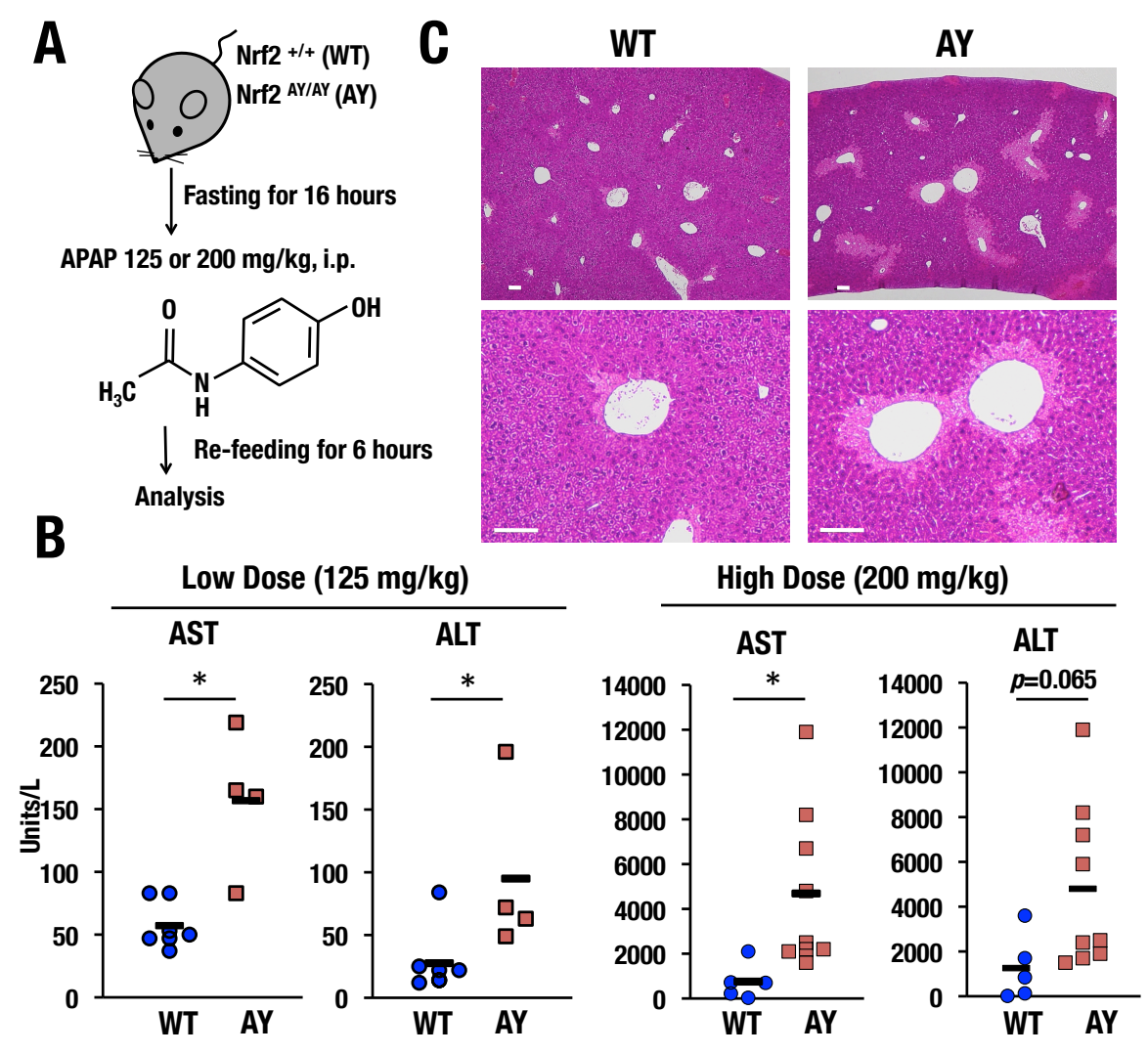

Figure 6 


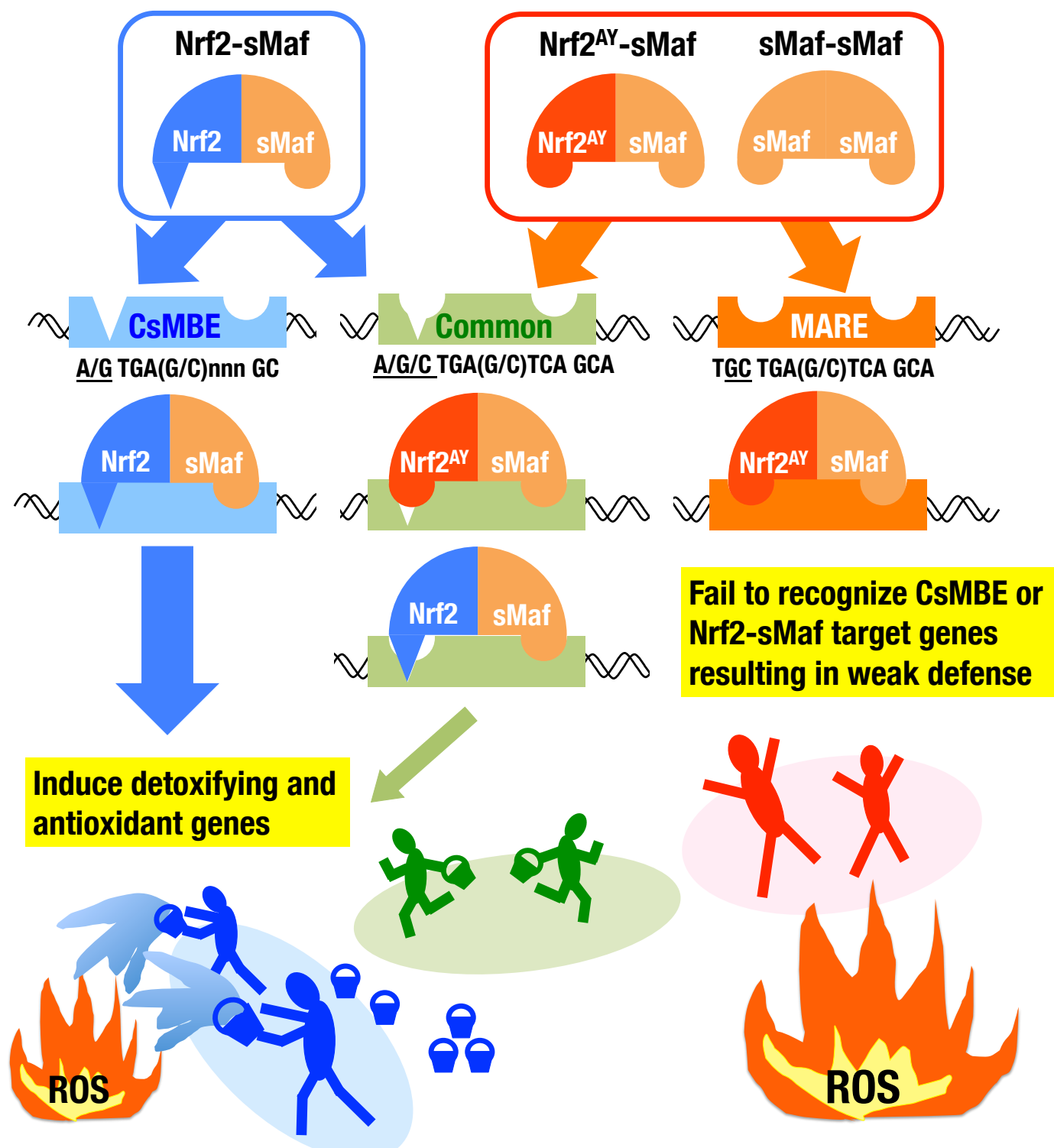

Figure 7 